\title{
Population II stars and the Spite plateau
}

\section{Stellar evolution models with mass loss ${ }^{\star}$}

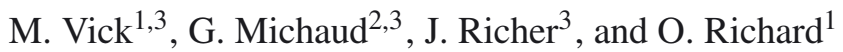 \\ ${ }^{1}$ LUPM, Université Montpellier II, CNRS CC072, Place E. Bataillon, 34095 Montpellier Cedex, France \\ e-mail: olivier . richard@univ-montp2 . fr \\ 2 LUTH, Observatoire de Paris, CNRS, Université Paris Diderot, 5 place Jules Janssen, 92190 Meudon, France \\ 3 Département de physique, Université de Montréal, Montréal, Québec, H3C 3J7, Canada \\ e-mail: [mathieu.vick; jacques.richer]@umontreal.ca, michaudg@astro.umontreal.ca
}

Received 12 November 2012 / Accepted 22 February 2013

\begin{abstract}
Aims. We aim to determine the constraints that observed chemical abundances put on the potential role of mass loss in metal poor dwarfs.

Methods. Self-consistent stellar evolutionary models that include all the effects of atomic diffusion and radiative accelerations for 28 chemical species were computed for stellar masses between 0.6 and $0.8 M_{\odot}$. Models with an initial metallicity of $Z_{0}=0.00017$ and mass loss rates from $10^{-15} M_{\odot} \mathrm{yr}^{-1}$ to $10^{-12} M_{\odot} \mathrm{yr}^{-1}$ were calculated. They were then compared to previous models with mass loss, as well as to models with turbulent mixing.

Results. For models with an initial metallicity of $[\mathrm{Fe} / \mathrm{H}]_{0}=-2.31$, mass loss rates of about $10^{-12} M_{\odot} \mathrm{yr}^{-1}$ lead to surface abundance profiles that are very similar to those obtained in models with turbulence. Both models have about the same level of agreement with observations of galactic-halo lithium abundances, as well as lithium and other elemental abundances from metal poor globular clusters such as NGC 6397. In this cluster, models with mass loss agree slightly better with subgiant observations of Li abundance than those with turbulence. Lower red giant branch stars instead favor the models with turbulence. Larger differences between models with mass loss and those with turbulence appear in the interior concentrations of metals.

Conclusions. The relatively high mass loss rates required to reproduce plateau-like lithium abundances appear unlikely when compared to the solar mass-loss rate. However the presence of a chromosphere on these stars justifies further investigation of the mass-loss rates.
\end{abstract}

Key words. diffusion - stars: abundances - stars: interiors - stars: Population II - stars: mass-loss

\section{Astrophysical context}

Population II stars can be used as tracers for exploring the universe's chemical evolution. Still, to correctly relate the chemical properties of the early Universe to the composition of the oldest observed stars, one must be able to map the evolution of their current abundances back to their original values. Doing so could help elucidate numerous questions pertaining to Big Bang nucleosynthesis, the nature of the first supernovae, and the age of the Universe.

The nearly constant Li abundance of most low-metallicity $([\mathrm{Z} / \mathrm{H}]<-1.5)$ halo field stars whose $T_{\text {eff }}$ is between $6300 \mathrm{~K}$ and $5500 \mathrm{~K}$ - otherwise known as the Spite plateau (Spite \& Spite 1982; Spite et al. 1984) - has puzzled astronomers for decades. Originally, many inferred that this plateau was representative of the Universe's original $\mathrm{Li}$ abundance, though it was promptly shown that this could not be the case (Michaud et al. 1984). Precise observations of the cosmic microwave background $^{1}$ have now led to a primordial lithium abundance ${ }^{2}$

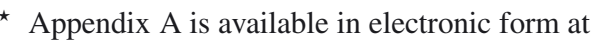
http: //wwW . aanda.org

1 From the Wilkinson Microwave Anisotropic Background (WMAP), see Spergel et al. (2007).

$2 A(\mathrm{Li})=\log [N(\mathrm{Li}) / N(\mathrm{H})+12]$.
}

of $A\left({ }^{7} \mathrm{Li}\right)=2.65_{-0.06}^{+0.05}$, or even higher, $A\left({ }^{7} \mathrm{Li}\right)=2.72_{-0.05}^{+0.05}($ Cyburt et al. 2003, 2008; Steigman 2007), while the lithium content in metal poor dwarfs, ranging from $A\left({ }^{7} \mathrm{Li}\right)=2.0$ to 2.4 is a factor of 2-3 lower (Bonifacio et al. 2007; Asplund et al. 2006; Charbonnel \& Primas 2005; Meléndez \& Ramírez 2004; Bonifacio 2002; Ryan et al. 1999).

Changing the standard Big Bang nucleosynthesis model is a possible explanation, though it is hypothetical (Ichikawa et al. 2004; Coc et al. 2004; Cumberbatch et al. 2007; Regis \& Clarkson 2012). Even though Population III stars may have destroyed some of the primordial lithium (Piau et al. 2006), this cannot explain the large discrepancy. This leads many to assume that the solution stems from within the stars themselves. Possible stellar explanations may include destruction via gravity waves (Talon \& Charbonnel 2004; Charbonnel \& Talon 2005), and rotational mixing (Vauclair 1988; Charbonnel et al. 1992; Pinsonneault et al. 1999), as well as settling via atomic diffusion (Deliyannis et al. 1990; Profitt \& Michaud 1991; Salaris et al. 2000; Richard et al. 2005). A higher Li content of subgiant (SG) stars compared to main-sequence (MS) stars in globular clusters (GC) such as NGC 6397 (Korn et al. 2007; Lind et al. 2009) favors a model in which lithium is not destroyed, but simply sunk below the photosphere prior to dredge-up. This result is, however, sensitive to the $T_{\text {eff }}$ scale used (González Hernández et al. 2009; Nordlander et al. 2012). 
Additional constraints may stem from claimed observations of ${ }^{6} \mathrm{Li}$ (Asplund et al. 2006), which, with a thermonuclear destruction cross section 60 times larger than the heavier isotope, would severely constrain the stellar destruction of ${ }^{7} \mathrm{Li}$. These observations remain very uncertain (Cayrel et al. 2007; Lind et al. 2012). Many recent studies have also claimed that below $[\mathrm{Fe} / \mathrm{H}] \simeq-2.5$, Li abundances lie significantly below the Spite plateau abundance and with increased scatter (Asplund et al. 2006; Aoki et al. 2009; Sbordone et al. 2010, 2012). Other observations suggest that there might instead be a lower plateau abundance for extremely metal poor (EMP) stars (Meléndez et al. 2010). The situation is complex and not currently understood in detail.

Although the surface convection zone (SCZ) of galactic Population II stars homogenizes abundances down to depths at which atomic diffusion is relatively slow, the long lifetimes of these stars, which reach the age of the Universe, allows for the effects of chemical separation to materialize at the surface. However, models that allow atomic diffusion to take place unimpeded lead to lithium underabundances that are too large at the hot end of the plateau (Michaud et al. 1984). By enforcing additional turbulent mixing below the SCZ, models of metal poor stars with gravitational settling and radiative accelerations are able to reproduce many observations of halo and globular cluster stars (Richard et al. 2002a,b, 2005; VandenBerg et al. 2002; Korn et al. 2006). These same models were also successful in explaining abundance anomalies in AmFm (Richer et al. 2000, 2001) and horizontal branch stars (Michaud et al. 2007, 2008).

In Vauclair \& Charbonnel (1995), gravitational settling is inhibited by unseparated mass loss at the constant rate of $\sim 3 \times 10^{-13} M_{\odot} \mathrm{yr}^{-1}$; this leads to near constant lithium abundances as a function of $T_{\text {eff }}$, even at the hot end of the plateau. However, these calculations, which are discussed in Sect. 4, did not include the effects of radiative accelerations, nor did they follow atomic diffusion in detail for elements heavier than $\mathrm{Li}$; therefore, further inspection is warranted.

In Vick et al. (2010, 2011), hereafter Papers I and II, stellar evolution models with mass loss were introduced and shown to reproduce observed surface abundance anomalies for many AmFm and chemically anomalous HAeBe binary stars. In Michaud et al. (2011) it was then shown that a mass loss rate compatible with the observed Sirius A mass loss rate leads to abundance anomalies similar to those observed on Sirius A. To further validate the model and to help constrain the relative importance of mass loss with respect to turbulent mixing in stars, the present mass loss models will be applied to Pop II stars and compared to models with turbulence from Richard et al. (2002b, 2005), which are able to reproduce observations of galactic halo stars, as well as of globular cluster stars on the subgiant and giant branches.

In the following analysis and in our calculations, mass loss is considered in non rotating stars, since in these stars it might be the only process competing with atomic diffusion. To our knowledge, mass loss has never been observed in Population II MS stars; therefore, mass loss rates will be constrained solely via surface abundance anomalies. In Sect. 2, the evolutionary calculations are presented, while the results are discussed in Sect. 3. In Sects. 4 and 5, the models with mass loss are compared to previous evolutionary models of metal poor dwarfs with mass loss, as well as to models with turbulence. In Sect. 6 results are compared to subgiant and giants both for models with turbulence and with mass loss. Conclusions follow in Sect. 7.

\section{Calculations}

The stellar evolution models were calculated as explained in Paper I and references therein. Atomic diffusion is computed self-consistently with radiative accelerations from Richer et al. (1998) and corrections for redistribution from Gonzalez et al. (1995) and LeBlanc et al. (2000). Chemical transport is computed for 28 chemical species (all those included in the OPAL database, Iglesias \& Rogers 1996, plus Li, Be, and B). The models are evolved from the pre-main-sequence (PMS) with the abundance mix prescribed in Richard et al. (2002b) for $Z_{0}=0.00017$ (or equally, $[\mathrm{Fe} / \mathrm{H}]=-2.31$ ). Most models were calculated with an initial helium fraction of 0.235 (corresponding to the $Y_{0}$ used for calculations for M 92 in VandenBerg et al. 2002). Since one of our major aims is to compare with similar models calculated with turbulence, this value was chosen to facilitate comparison (since it is the same as in Richard et al. 2002b, 2005). Some models were also calculated with $Y_{0}=$ 0.2484, which was derived from WMAP observations (Cyburt et al. 2003) and is discussed in Sect. 3.3.1 of Richard et al. (2005). As shown in Fig. 4 of VandenBerg et al. (1996) and Fig. 5 of Richard et al. (2005), respectively, this small change in initial helium fraction has little effect on the luminosity-versusage relation ${ }^{10}$ or on lithium isochrones. With respect to the solar abundance mix used in Papers I and II for Population I stars, the relative abundances of alpha elements are increased as appropriate for Population II stars (VandenBerg et al. 2000). The introduction of mass loss and its impact on transport is outlined in Paper I.

The convection zone boundary is determined by the Schwarzchild criterion with energy flux in the convection zone computed with Böhm-Vitense (1992) mixing length formalism. Abundances are assumed to be completely mixed within convection zones. No extra mixing is enforced outside of convection zones. The effects of atomic diffusion materialize as soon as radiative zones appear as the models evolve toward the MS. These effects become more important as the radiative zone expands toward the surface, where atomic diffusion becomes more efficient, and the convection zone becomes less massive, thereby reducing the dilution of anomalies.

The unseparated ${ }^{3}$ mass loss rates considered range from $10^{-15}$ to $10^{-12} M_{\odot} \mathrm{yr}^{-1}$. Owing to uncertainties related to the nature of winds for Population II stars (see also discussion in Sect. 4.2 of Paper I), we have chosen to limit our investigation to unseparated winds in order to avoid introducing additional adjustable parameters.

In order to fully constrain the effects of mass loss, we have chosen to use the same boundary condition (Krishna-Swamy 1966) and mixing length parameter, $\alpha=2.096$, as in Papers I and II (see also Sect. 2 of Paper I and VandenBerg et al. 2008). Most models in Richard et al. (2002a,b, 2005) were computed with the Eddington gray atmosphere surface boundary condition (see also Turcotte et al. 1998). The main consequence is to change the value of $\alpha$. In both cases, the mixing length is calibrated using the Sun so that very little difference remains in solar type models. Therefore, though the different boundary conditions might lead to a different evolution of SCZ depths and masses (see Fig. 9 of Richard et al. 2002b), the remaining slight difference does not play a role in models with turbulence since the separation that modulates surface abundances usually occurs deeper. As shown in Sect. 3, the same is true for the models with

3 In this context, unseparated refers to the abundances in the wind being the same as in the photosphere. 

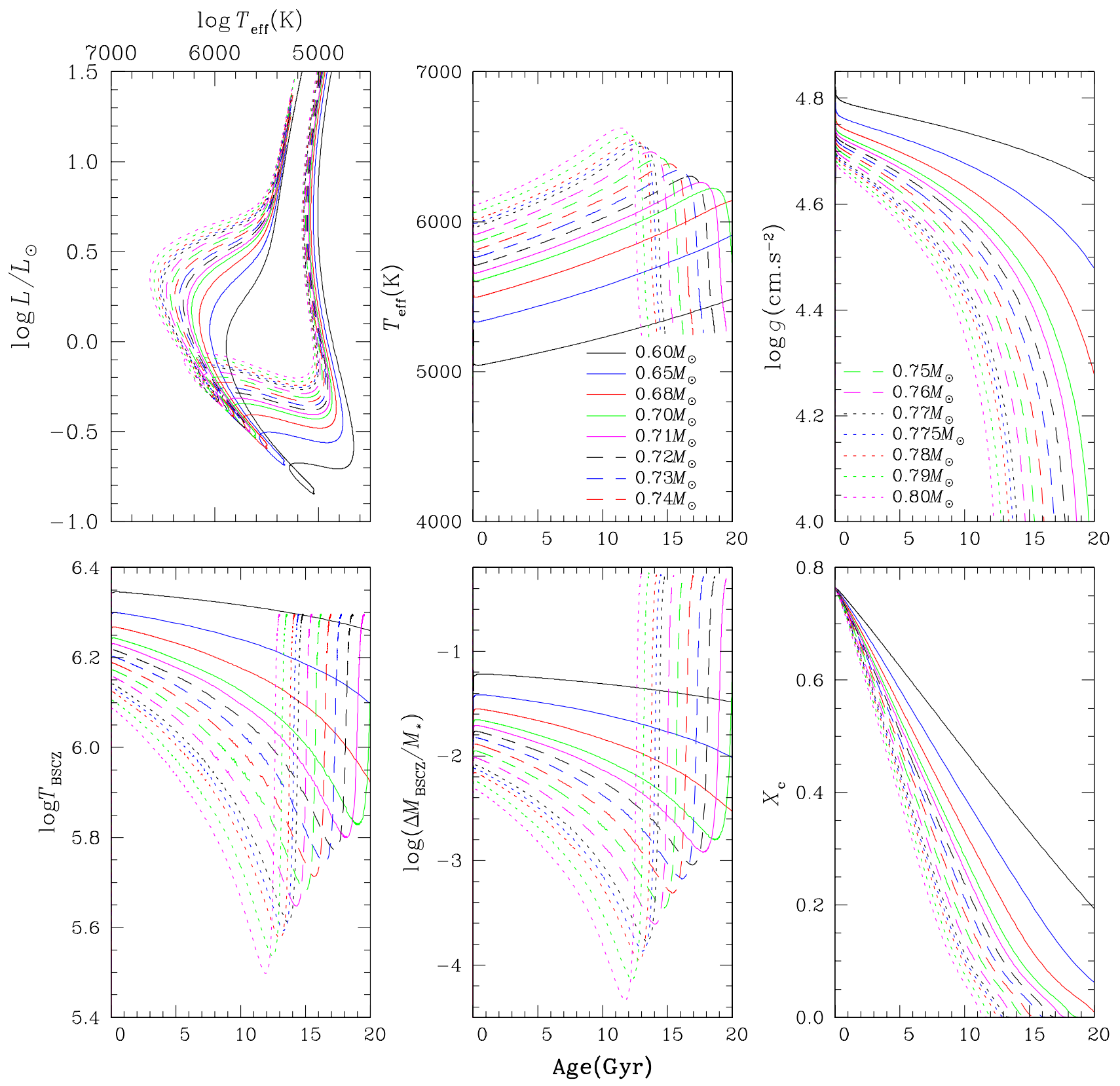

Fig. 1. The Hertzprung-Russel diagram, as well as the evolution of $T_{\mathrm{eff}}, \log g$, the temperature at the base of the SCZ (TSCZ), the outer mass at the base of the $\operatorname{SCZ}\left(\Delta M_{\mathrm{BSCZ}}\right)$, and the mass fraction of hydrogen in the core $\left(X_{\mathrm{c}}\right)$ for stars of $0.6-0.8 M_{\odot}$ with $Z_{0}=0.00017$ and $\dot{M}=10^{-12} M_{\odot} \mathrm{yr}^{-1}$.

mass loss presented in this paper since separation also occurs deeper than the bottom of the convection zone.

\section{Evolutionary models}

Evolutionary models of masses ranging from 0.6 to $0.8 M_{\odot}$, an interval of masses that spans the Spite plateau, are shown in a Hertzprung-Russel diagram (H-R) in Fig. 1. All the models have an initial metallicity of $Z_{0}=0.00017$, and a mass loss rate of $10^{-12} M_{\odot} \mathrm{yr}^{-1}$. Figure 1 also shows the evolution of $T_{\text {eff }}$, gravity, the depth (in mass, $\Delta M_{\mathrm{BSCZ}}$, and in temperature, $T_{\mathrm{BSCZ}}$ ) of the bottom of the SCZ, and the central $\mathrm{H}$ mass fraction. The 0.79 and $0.77 M_{\odot}$ models are respectively at the MS turnoff at $12 \mathrm{Gyr}$ and $13.5 \mathrm{Gyr}$, the assumed lower and upper limits of the age of halo dwarfs.

For all models, the variations in surface convection zone depth have an impact on surface-abundance evolution since the efficiency of atomic diffusion varies with depth. Throughout the MS, $\Delta M_{\mathrm{BSCZ}}$ decreases until $\mathrm{H}$ is exhausted in the stellar core (i.e. until turnoff). Although this and other parameters depend on the mass loss rate, the effect is slight (see Fig. 2). Just before the MS turnoff, there is a $\sim 125 \mathrm{~K}$ difference in $T_{\text {eff }}$ between the $0.8 M_{\odot}$ model with diffusion only and the $0.80 \mathrm{~W} 1 \mathrm{E}-12 \mathrm{model}^{4}$, while the difference falls to about $15 \mathrm{~K}$ between the diffusion only and $0.80 \mathrm{~W} 1 \mathrm{E}-13$ models. The temperature and mass at the bottom of the SCZ are related to both the $\mathrm{He}$ and Fe concentrations in the SCZ. For instance, the temperature is lowest when both $\mathrm{He}$ and Fe have a small abundance at turn-off in the $0.80 \mathrm{~W} 1 \mathrm{E}-13$ model. The temperature is highest in the models with largest $\mathrm{He}$ and $\mathrm{Fe}$ abundances (and highest mass loss rate) and intermediate in the diffusion only model where the

\footnotetext{
$40.80 \mathrm{~W} 1 \mathrm{E}-12$ refers to a $0.80 M_{\odot}$ model with a mass loss rate of $1 \times$ $10^{-12} M_{\odot} \mathrm{yr}^{-1}$.
} 

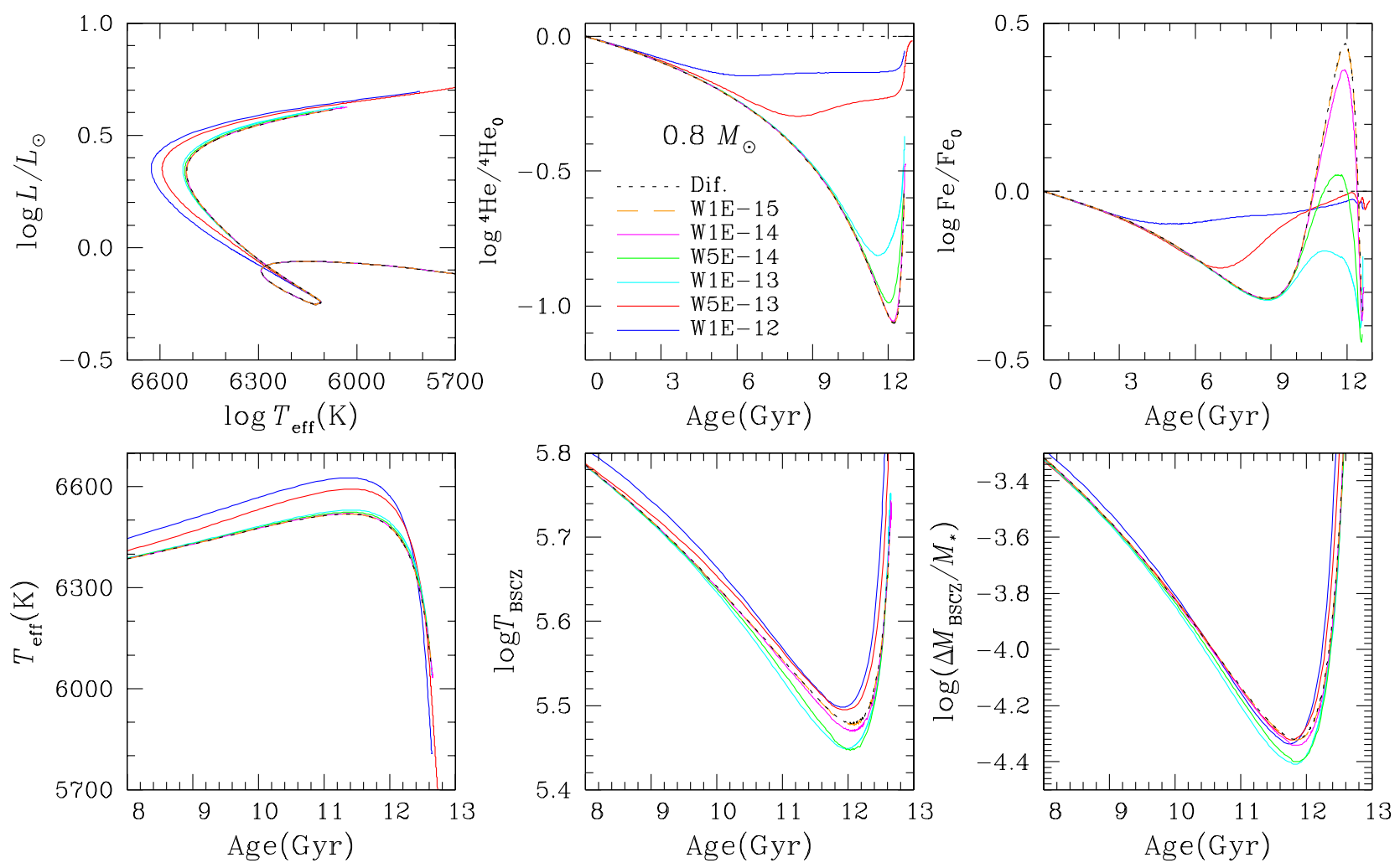

Fig. 2. The impact of mass loss on the evolution of the depth (in temperature and mass) of the SCZ in $0.8 M_{\odot}$ models with atomic diffusion only, and with mass loss rates from $10^{-15} M_{\odot} \mathrm{yr}^{-1}$ to $10^{-12} M_{\odot} \mathrm{yr}^{-1}$. Upper row: HR diagram and the He and Fe surface abundances. Lower row: zoom on the $T_{\text {eff }}$, and on the mass and temperature at the bottom of the SCZ.

low He abundance is partly canceled by the high Fe abundance. The iron abundance at turn-off is largest $(+0.47 \mathrm{dex})$ in the model without mass loss and decreases progressively to -0.2 dex as the mass loss rate increases to $10^{-13} M_{\odot} \mathrm{yr}^{-1}$ and then is very nearly normal in the larger mass loss cases. The effect on stellar age at the turnoff, or equivalently, on the stellar lifetime, is less than the line thickness in the plots. While $\Delta M_{\mathrm{BSCZ}} / M_{*}$ varies by a factor of $100\left(\log \Delta M / M_{*}\right.$ goes from $\sim-2.3$ to -4.3$)$ on the MS for a $0.80 M_{\odot}$ model (Fig. 1), the maximum effect of adding mass loss is below 0.08 dex.

\subsection{Radiative accelerations, mass loss rate, and internal abundance variations}

On very short time scales, convection homogenizes abundances from the bottom of the SCZ to the stellar surface; therefore, the competition between radiative acceleration $\left(g_{\mathrm{rad}}\right)$ and gravity is only important below the surface convection zone. In Fig. 3, the internal variations of $g_{\mathrm{rad}}$, velocity, and mass fraction are shown for $\mathrm{Li}, \mathrm{Mg}, \mathrm{Ca}, \mathrm{Ti}, \mathrm{Fe}$, and $\mathrm{Ni}$ in the $0.80 \mathrm{~W} 1 \mathrm{E}-12$ model $^{5}$. Having $g_{\text {rad }}$, velocity, and mass fraction aligned for a given element facilatates the discussion of the link between those quantities. Figures for $g_{\mathrm{rad}}$ and mass fraction of all elements are shown in Appendix A; they allow to show the systematic variations of those quantities. The changes in $g_{\text {rad }}$ over time are due principally to structure changes, since the abundances in Population II stars with $[\mathrm{Fe} / \mathrm{H}]_{0}=-2.31$ are never large enough to cause saturation ${ }^{6}$.

\footnotetext{
5 This mass was chosen to facilitate comparison with the models and figures from Richard et al. (2002b).

6 The mass loss rate also has little to no effect on $g_{\text {rad }}$ for this same reason, and because its effects on the structure are very small (see Fig. 2).
}

The internal variations of an element's $g_{\text {rad }}$ can be related to its ionization state as may be seen in the online Appendix A; $\mathrm{H}$-like electronic configurations lead to local maxima, while $\mathrm{He}$ like configurations lead to local minima. As we move toward elements of higher atomic number, the H-like state is encountered at greater depths. Carbon for instance, is in an H-like configuration at $\log \Delta M / M_{*} \sim-3$, while for $\mathrm{Si}$, this occurs near $\log \Delta M / M_{*} \sim-1$. Some elements, including $\mathrm{Fe}$ and $\mathrm{Ni}$, never quite reach the $\mathrm{H}$-like state ${ }^{7}$. Unlike the $\mathrm{H}$-like maximum, the second local $g_{\text {rad }}$ maximum, which is clearly distinguishable for all elements heavier than $\mathrm{S}$, is usually greater than or equal to gravity. This $g_{\text {rad }}$ increase corresponds to configurations between F-like and Li-like.

For many elements, including $\mathrm{He}, \mathrm{Li}$, and $\mathrm{O}$, the radiative acceleration is always smaller than gravity. For a mass loss rate of $10^{-12} M_{\odot} \mathrm{yr}^{-1}$, these elements are underabundant (bottom row of Fig. 3) throughout the star except close to the center. Other elements such as Be, $\mathrm{Mg}$, and $\mathrm{Fe}$ have local $g_{\text {rad }}$ maxima just below the SCZ, which, at $11664 \mathrm{Myr}$, causes their surface abundances to be larger than just below the convection zone. All abundance variations caused by atomic diffusion are smaller than $0.2 \mathrm{dex}$ (in contrast to the ones caused by thermonuclear reactions which are encountered near and around the stellar core for $Z<8$ ). For this mass loss rate, all elements are underabundant below the SCZ, and down to about $\log \Delta M / M_{*} \sim-0.5$. What varies most rapidly is the location of the bottom of the SCZ. The time variation of interior concentrations is continuous and relatively small. Larger variations are linked to local extrema of $g_{\mathrm{rad}}$. For instance $X(\mathrm{Fe})$

7 To be more precise, in the stellar core, these elements arrive at an equilibrium between multiple ionization states, including the hydrogenic one. 
M. Vick et al.: Population II stars and the Spite plateau
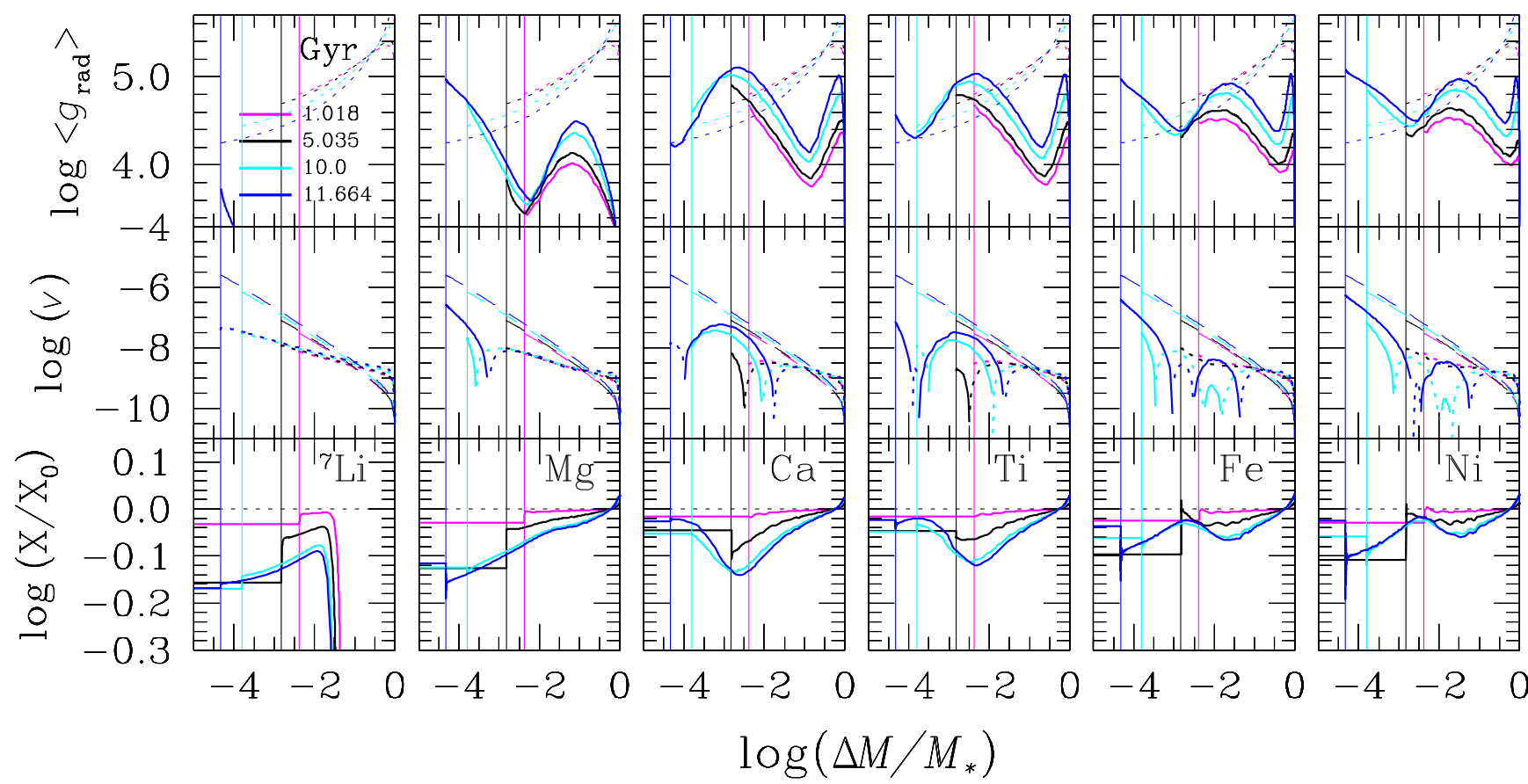

Fig. 3. Upper row: radiative accelerations (solid lines) and gravity (dotted lines); center row: wind velocities (dashed lines) and diffusion velocities toward the exterior (solid lines) and toward the interior (dotted lines); lower row: interior concentrations as a fraction of original ones. These quantities are shown at four different ages for a $0.8 M_{\odot}$ model with $\dot{M}=10^{-12} M_{\odot} \mathrm{yr}^{-1}$. The vertical lines indicate the position of the bottom of the SCZ. The ages are identified in the figure.
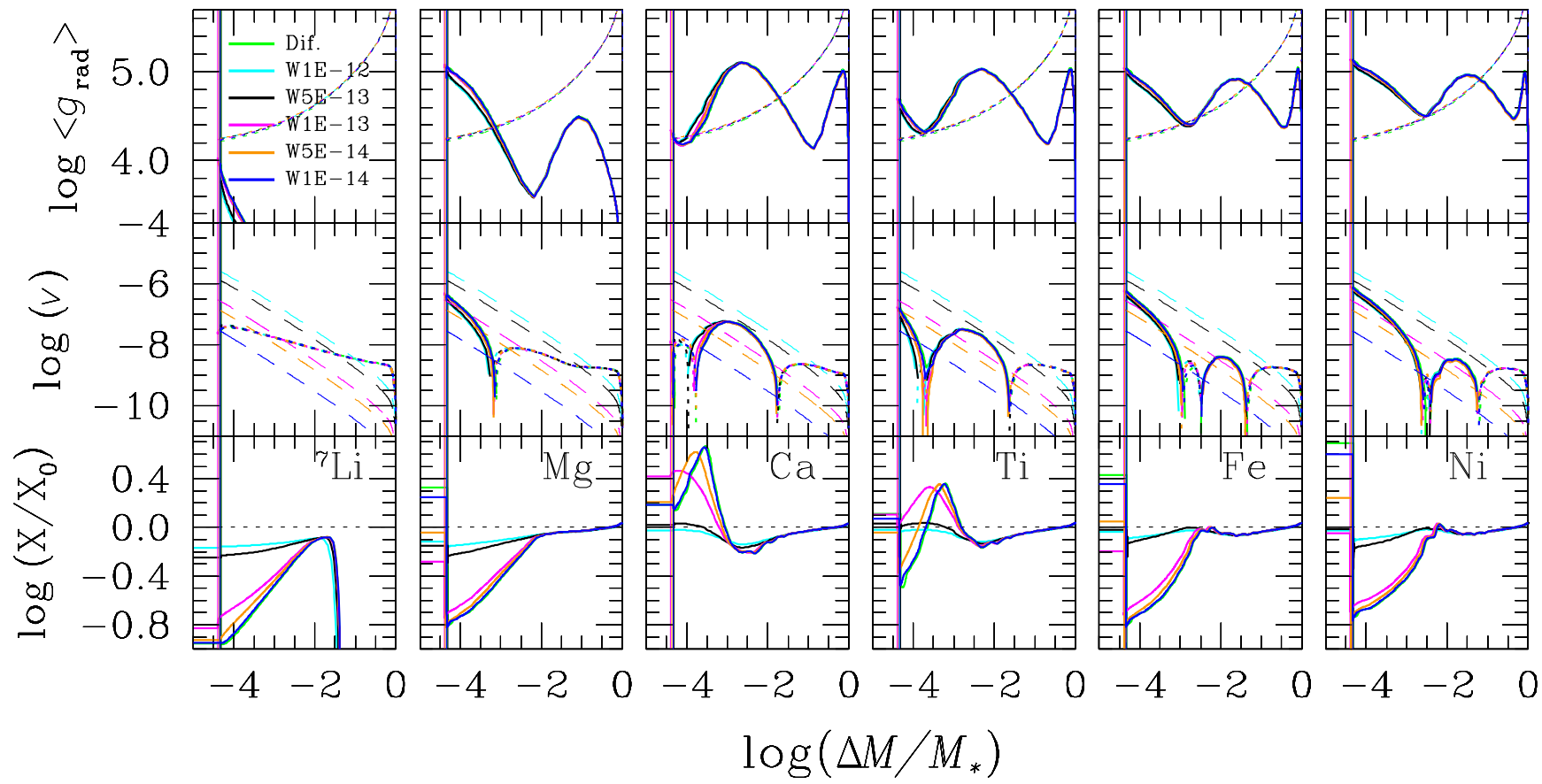

Fig. 4. The impact of mass loss rate on internal abundance profiles for $0.80 M_{\odot}$ models. A model without mass loss and models with five mass loss rates from $10^{-14} M_{\odot} \mathrm{yr}^{-1}$ to $10^{-12} M_{\odot} \mathrm{yr}^{-1}$ are shown and are identified in the figure. The internal concentration profiles are shown just before turnoff near 11.5 Gyr. Atomic diffusion affects mainly the outer $10^{-2}$ of the stellar mass. Conventions are similar to those of Fig. 3.

has a local maximum at $\log \Delta M / M_{*} \sim-3$ where $g_{\mathrm{rad}}(\mathrm{Fe})$ has a minimum and the diffusion velocity is inwards. These effects will be discussed below in relation to the effect of varying the mass loss rate. Below $\log \Delta M / M_{*} \sim-0.5$, elements heavier than $\mathrm{O}$ become slightly overabundant due to gravitational settling. Therefore, for these elements, there are underabundances of up to a factor of 1.6 over the outer half of the stellar mass and overabundances of a few percent over the inner half.
For models with lower mass loss rates, internal abundance variations become much greater (Fig. 4). In the $0.80 M_{\odot}$ model with a $10^{-13} M_{\odot} \mathrm{yr}^{-1}$ mass loss rate, $\mathrm{Ca}$ and Ti are locally overabundant by a factor of up to $\sim 3$, while ${ }^{4} \mathrm{He},{ }^{7} \mathrm{Li}, \mathrm{Mg}$, and $\mathrm{Fe}$ become -0.8 dex underabundant. These variations can be linked directly to variations in $g_{\mathrm{rad}}$. For example, the Ni underabundance that begins at $\log \Delta M / M_{*} \sim-2.5$, and reaches up to the BSCZ, is caused by the increase in its $g_{\mathrm{rad}}$ over the same 


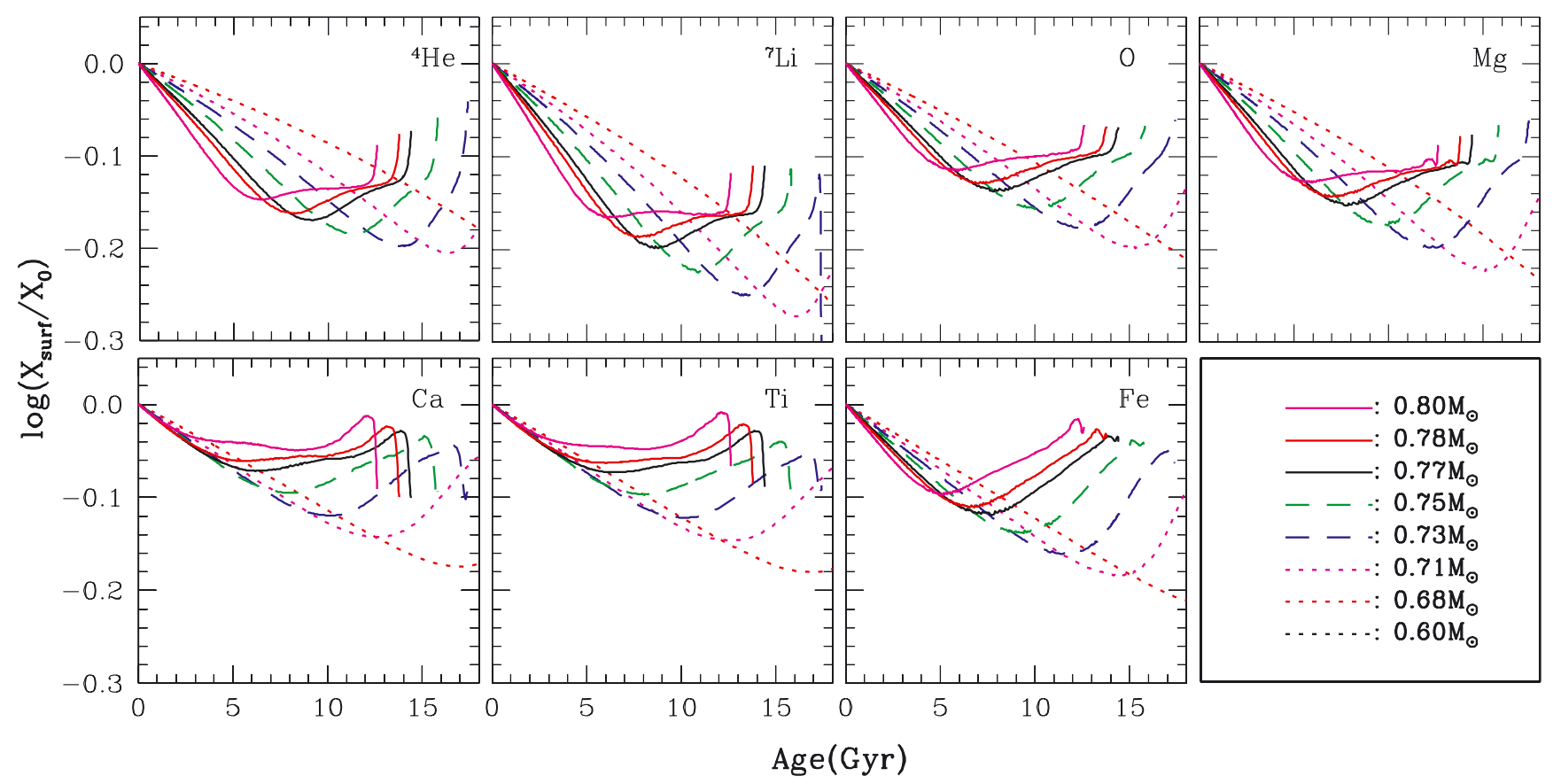

Fig. 5. Evolution of surface abundance anomalies for selected stellar masses and elements with a mass loss rate of $10^{-12} M_{\odot} \mathrm{yr}^{-1}$.

depth interval. In this region, $\mathrm{Ni}$ is pushed into the SCZ, thus causing the $\mathrm{Ni}$ abundance to be relatively larger at the surface and to decrease below the SCZ. In contrast, $\mathrm{Ca}$ is overabundant near $\log \Delta M / M_{*}=-3.5$ and above, since $g_{\text {rad }}(\mathrm{Ca})$ has a local maximum just below this depth.

In fact, when mass loss is added, one may distinguish two types of internal solutions: those dominated by atomic diffusion and those dominated by mass loss (see also Sect. 5.1.1 of Paper I). For any given model with mass loss, an element's internal abundance solution is determined by the mass loss rate wherever the associated velocity, $v_{\text {wind }}$, is greater in amplitude than the settling velocity $v_{\text {sett }}$. For example, in Fig. 4 , one first notes that the depth at which $\left|v_{\text {wind }}\right| \simeq\left|v_{\text {sett }}\right|$, which we call the point of separation, depends on the element and on the stellar age (through structure changes). For the $0.80 \mathrm{~W} 1 \mathrm{E}-12$ model, mass loss dominates the internal solution of ${ }^{7} \mathrm{Li}$ from the surface down to $\log \Delta M / M_{*} \sim-1.2$, while this occurs nearer $\log \Delta M / M_{*} \sim-0.9$ for Ni. As discussed in Sect. 5.1.1 of Paper I, the consequence is that local abundances above the point of separation are not determined uniquely by the local $g_{\text {rad }}$, but instead adjust in order to conserve the flux arriving from just below the point of separation. If the wind-dominated solution extends down to $\Delta M \equiv \dot{M} \times t$, the surface abundance reflects $g_{\mathrm{rad}} / g$ at $\Delta M$. If we come back to the previous example, at $10 \mathrm{Gyr}-$ or $10^{10} \mathrm{yr}-$ and for a mass loss rate of $10^{-13} M_{\odot} \mathrm{yr}^{-1}$, the surface reflects $g_{\mathrm{rad}} / g$ at $\sim 10^{-3} M_{*}$.

In general, a higher mass loss rate has the effect of reducing internal anomalies. For example, a mass loss rate of $10^{-12} M_{\odot} \mathrm{yr}^{-1}$ reduces the $\mathrm{Li}$ and $\mathrm{Mg}$ anomalies below the convection zone by $\sim 0.6$ dex (Fig. 4). However, the abundance anomalies of, for instance, $\mathrm{Fe}$ do not always have a monotonous variation with mass loss rate. As one reduces the mass loss from $10^{-12} M_{\odot} \mathrm{yr}^{-1}$, the Fe abundance in and below the SCZ first decreases and is 0.6 dex smaller below the SCZ in the $10^{-13} M_{\odot} \mathrm{yr}^{-1}$ model. In the SCZ it is 0.2 dex smaller. However, in the model with a mass loss rate of $10^{-13} M_{\odot} \mathrm{yr}^{-1}$, the surface $\mathrm{Fe}$ abundance is $0.35 \mathrm{dex}$ larger than the original abundance, and without mass loss, it is 0.4 dex larger than the original one ${ }^{8}$. This surprising behavior is related to the minimun of $g_{\mathrm{rad}}(\mathrm{Fe})$ at $\log \Delta M / M_{*} \sim-3$. For a mass loss rate of $10^{-13} M_{\odot} \mathrm{yr}^{-1}$, the wind carries away the surface $\mathrm{Fe}$ pushed by $g_{\mathrm{rad}}(\mathrm{Fe})$ above $\log \Delta M / M_{*} \sim-3$ since in one Gyr, it carries $\log \Delta M / M_{*} \sim-4$ and the SCZ is slightly less than $\log \Delta M / M_{*} \sim-4$. The $\mathrm{Fe}$ flux that crosses the region where the Fe diffusion velocity is negative, at $\log \Delta M / M_{*} \sim-3$, is reduced, leading to the observed underabundances. The $10^{-14} M_{\odot} \mathrm{yr}^{-1}$ wind carries a lower Fe mass from the outer region and reduces the surface $\mathrm{Fe}$ by only 0.05 dex compared to the no-mass-loss model.

On the other hand, $\mathrm{Ca}$ in the $0.80 \mathrm{~W} 1 \mathrm{E}-13$ model in Fig. 4 has a larger surface overabundance than in the no-mass-loss model. This is because the $\mathrm{Ca}$ abundance must adjust to carry the flux strongly pushed upwards at $\log \Delta M / M_{*} \sim-3$ where $g_{\mathrm{rad}}(\mathrm{Ca})$ is much larger than gravity. In the $10^{-14} M_{\odot} \mathrm{yr}^{-1}$ model, the wind velocity is too weak to carry the flux when the diffusion velocity is toward the interior, and there is a sharp peak of $X(\mathrm{Ca})$ at $\log \Delta M / M_{*}=-3.5$. The profile of the concentration is clearly linked to the profile of the downward oriented diffusion velocity, from $\mathrm{Mg}$ to $\mathrm{Fe}$.

\subsection{Surface abundance variations}

For a given metallicity, surface abundances depend on stellar mass and age as well as on mass loss rate. The age and stellar mass dependance are demonstrated in Fig. 5, which shows the evolution of surface abundances for selected models with a mass loss rate of $10^{-12} M_{\odot} \mathrm{yr}^{-1}$. First, for this mass loss rate, all elements are underabundant throughout MS evolution, and until dredge up advects layers which are affected by thermonuclear reactions. The underabundance amplitudes increase as stellar mass decreases, since settling becomes more important for models with longer lifetimes. Over a large fraction of the stellar lifetime, the overall shape of the surface abundance solution is

\footnotetext{
8 We have verified that the $10^{-15} M_{\odot} \mathrm{yr}^{-1}$ model is the same as the nomass-loss model. See Fig. 2.
} 
M. Vick et al.: Population II stars and the Spite plateau

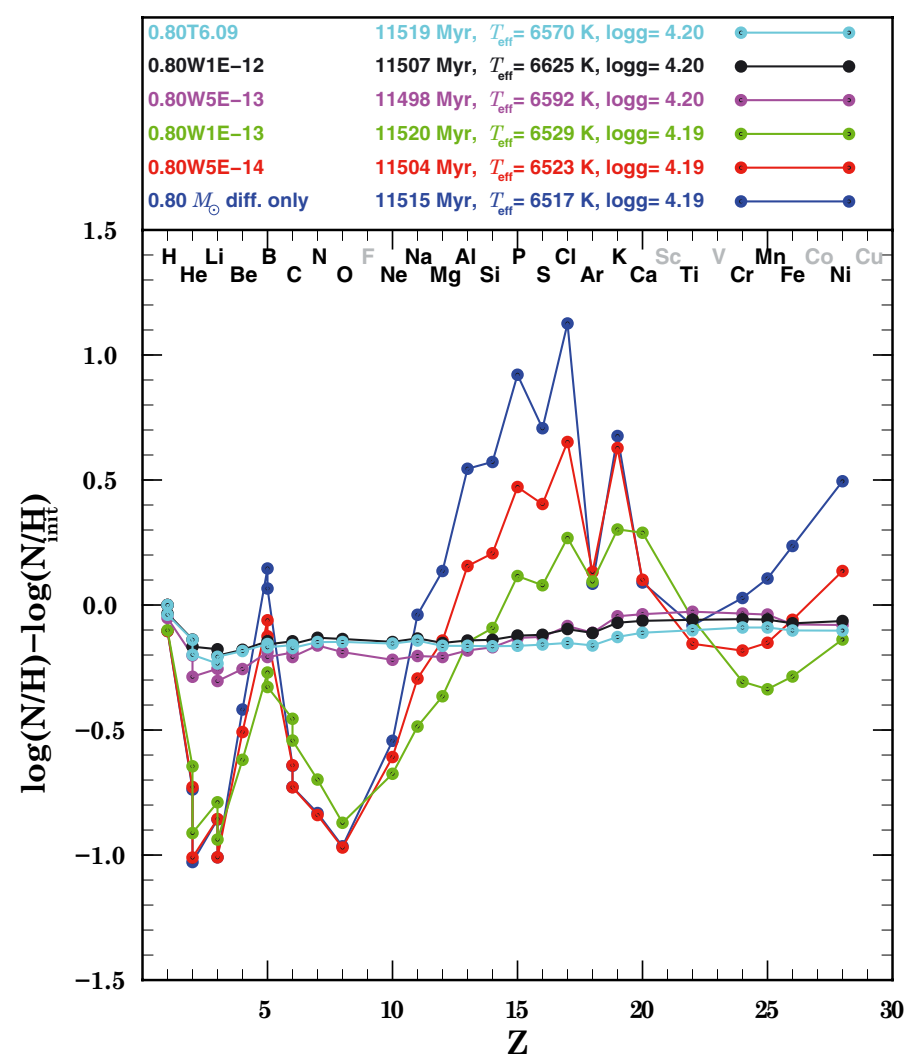

Fig. 6. Surface abundance anomalies for $0.8 M_{\odot}$ models with and without mass loss as well as with the T6.09 turbulent parametrization. At 11.5 Gyr, these models are just before turnoff.

quite similar for all masses since the overall shape of $g_{\text {rad }}$ does not vary significantly over this mass interval. See Fig. 2 for the effect of varying the mass loss rate on the He and Fe abundances.

At a given age, the mass loss rate affects the surface abundance of the various elements differently, since supported elements are expelled through the surface, while sinking elements encounter advection by $v_{\text {wind }}$. For $0.80 M_{\odot}$ models near turnoff (Fig. 6), a mass loss rate of $10^{-13} M_{\odot} \mathrm{yr}^{-1}$ reduces He, $\mathrm{Li}$, and $\mathrm{O}$ underabundances obtained in the diffusion only model by a little more than 0.1 dex, while also reducing overabundances of $\mathrm{Cl}$ and $\mathrm{P}$ by about $0.8 \mathrm{dex}$. It is then clear that for this mass loss rate, the wind's effect is greater for overabundant (i.e. supported) elements, since the settling velocity still dominates for unsupported elements. For yet other elements the abundance variations are more complex: the overabundances of $\mathrm{Fe}$ and $\mathrm{Ni}$ become underabundances, while $\mathrm{Ca}$ and $\mathrm{Ti}$ become more overabundant for a short period of time. For $\mathrm{Ca}$ and $\mathrm{Ti}$, this is because $v_{\text {wind }}$ has an effect just below the SCZ, where $g_{\text {rad }} \simeq g$ (Fig. 4); therefore, instead of accumulating below the SCZ, they are pushed into it. Similarly, mass loss advects the layers just below the SCZ, where $\mathrm{Fe}$ and $\mathrm{Ni}$ abundances are smallest, into the SCZ (see Figs. 4 and A.3). For the two higher mass loss rates, the surface anomalies are reduced to factors mostly below about 0.2 dex. While the $0.80 \mathrm{~W} 1 \mathrm{E}-12$ has generalized underabundances, the $0.80 \mathrm{~W} 5 \mathrm{E}-13$ model has small overabundances of elements from $\mathrm{K}$ to $\mathrm{Mn}$ (see Fig. A.3).

\subsubsection{Surface lithium abundances}

Figure 7 shows lithium and iron isochrones for the diffusion only model, for models with mass loss rates of $10^{-12} M_{\odot} \mathrm{yr}^{-1}$ and $5 \times 10^{-13} M_{\odot} \mathrm{yr}^{-1}$, as well as for T6.09 models ${ }^{9}$ at $12 \mathrm{Gyr}$ and 13.5 Gyr. To simplify the discussion only the effect of mass loss is considered in this section. The T6.09 isochrones that are from Richard et al. (2002b) are discussed in Sect. 5 and where the effects of mass loss and turbulence are compared and the meaning of T6.09 is briefly described.

In the diffusion only model, large $\mathrm{Fe}$ and $\mathrm{Li}$ anomalies are obtained around turnoff: lithium decreases by a factor of up to 10 at $12 \mathrm{Gyr}$, while $\mathrm{Fe}$ becomes overabundant by a factor of 5. For the mass loss rate of $5 \times 10^{-13} M_{\odot} \mathrm{yr}^{-1}$, the maximum lithium underabundance is reduced to -0.35 dex, whereas $\mathrm{Fe}$ overabundances are transformed into underabundances by factors below $-0.25 \mathrm{dex}$. For the $10^{-12} M_{\odot} \mathrm{yr}^{-1}$ model, the maximum amplitudes are further reduced. While the anomalies in the diffusion only model near turnoff are much smaller at $13.5 \mathrm{Gyr}$ than at $12 \mathrm{Gyr}$, this effect is not as important for lithium, and is unnoticeable for both models with mass loss.

Below $\sim 6000 \mathrm{~K}$, the lithium isochrones with and without mass loss differ only slightly for preturnoff stars - with lithium depletions between -0.12 and -0.14 dex near $T_{\text {eff }}=5400 \mathrm{~K}$ - since the SCZ is too deep for mass loss to have an effect. Though all three isochrones have decreasing lithium abundances for models hotter than $\sim 6000 \mathrm{~K}$, the underabundance amplitude decreases as the mass loss rate increases. At $12 \mathrm{Gyr}$, the lithium abundance begins increasing again near $6200 \mathrm{~K}$ and $6400 \mathrm{~K}$ for the models with $10^{-12} M_{\odot} \mathrm{yr}^{-1}$ and $5 \times 10^{-13} M_{\odot} \mathrm{yr}^{-1}$ respectively. This is due to SCZ retraction, which exposes layers for which $v_{\text {wind }}$ is dominant.

The diffusion only lithium isochrones do not exhibit plateaulike behavior. On the other hand, the mass loss isochrones are much flatter: the $5 \times 10^{-13} M_{\odot} \mathrm{yr}^{-1}$ model has a scatter of about $\sim 0.1$ dex and the $10^{-12} M_{\odot} \mathrm{yr}^{-1}$ model has a scatter of about $\sim 0.05$ dex with respective plateau average values of about -0.3 dex and -0.2 dex below the initial ${ }^{7} \mathrm{Li}$ abundance.

\section{Comparison to previous calculations with mass loss}

In previous evolutionary calculations for Population II stars with mass loss, Vauclair \& Charbonnel (1995, VC95) found that both mass loss rates of $10^{-12.5}\left(\simeq 3 \times 10^{-13}\right)$ and $10^{-12} M_{\odot} \mathrm{yr}^{-1}$ lead to lithium abundances that increase monotonously (by at most 0.5 dex for the lower mass loss rate) with $T_{\text {eff }}$ for six models ranging from 0.60 to $0.80 M_{\odot}$. They suggested that the higher mass loss rate completely wipes out the effects of atomic diffusion, and leads to a primordial lithium abundance of $A(\mathrm{Li})=2.5 \pm 0.1$, which they determined from the surface abundance of their hottest model.

Unfortunately, a detailed comparison to their results is impossible, since, even without mass loss, the stellar models are too different. These differences include $Y_{0}, Z_{0}, \alpha_{\mathrm{MLT}}$, the surface boundary condition, and the diffusion of metals. As a result,

9 The parameters specifying turbulent transport coefficients are indicated in the name assigned to the model. For instance, in the T6.09D400-3 model, the turbulent diffusion coefficient, $D_{\mathrm{T}}$, is 400 times greater than the He atomic diffusion coefficient at $\log T_{0}=$ 6.09 and varying as $\rho^{-3}$, or

$D_{\mathrm{T}}=400 D_{\mathrm{He}}\left(T_{0}\right)\left[\frac{\rho}{\rho\left(T_{0}\right)}\right]^{-3}$

To simplify writing, T6.09 is also used instead of T6.09D400-3 since all models discussed in this paper have the D400-3 parametrization. The 0.80T6.09 model is a $0.80 M_{\odot}$ model with T6.09 turbulence. 
A\&A 552, A131 (2013)

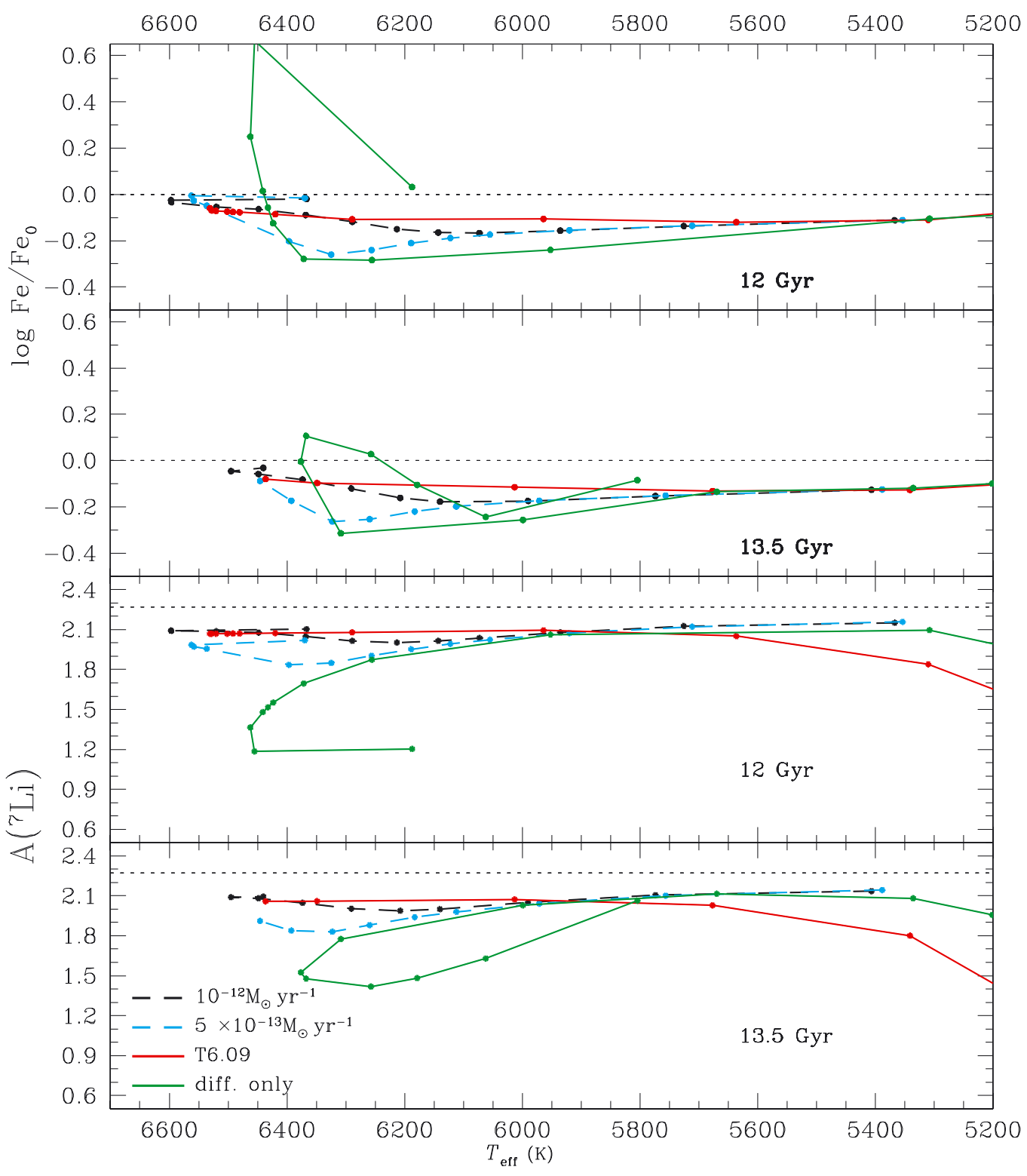

Fig. 7. Lithium and iron surface abundance isochrones at $12 \mathrm{Gyr}$ and $13.5 \mathrm{Gyr}$ for models with and without mass loss as well as the T6.09 models with turbulence but no mass loss. The straight line segments link models (dots) calculated with the same physics. The horizontal dotted line at $A\left({ }^{7} \mathrm{Li}\right)=2.27$ indicates the initial lithium abundance for all models. The model masses are not the same for every isochrone.

the SCZ evolution is very different. For instance, by comparing the internal lithium profile of the diffusion only model in Fig. 8 with the one from Fig. 4 of Vauclair \& Charbonnel (1995), one sees that at $10 \mathrm{Gyr}$, the SCZ (i.e. the flat part of the profile for both solutions) is about four times more massive in their models. Furthermore, their $0.75 M_{\odot}$ models are still on the MS (with their $T_{\text {eff }}$ still increasing) at $18 \mathrm{Gyr}$, while our $0.75 M_{\odot}$ models have already evolved toward the subgiant branch at $15 \mathrm{Gyr}$. The difference in $Y_{0}$ is not large enough to explain this significant discrepancy ${ }^{10}$.

That being said, some general results can still be compared. For instance, at $10 \mathrm{Gyr}$, VC95 found that the surface lithium abundance for the $10^{-12.5} M_{\odot} \mathrm{yr}^{-1}$ model was 0.12 dex larger than in the $10^{-12} M_{\odot} \mathrm{yr}^{-1}$ model, since the stronger mass loss rate

\footnotetext{
10 Though not shown, this was verified by evolving models with $Y_{0}=0.2484$, whose lower $\mathrm{H}$ mass fraction in the core led to their passing turnoff only $1 \mathrm{Gyr}$ earlier than models with $Y_{0}=0.2352$. The models from Vauclair \& Charbonnel (1995) have $Y_{0}=0.2403$.
}

reaches into the lithium burning layers. Our models also suggest that the strongest wind drags some material from the lithium depleted layers outward. Indeed, from Fig. 8, the wind velocity for the $10^{-12} M_{\odot} \mathrm{yr}^{-1}$ model at $10 \mathrm{Gyr}$ dominates the settling velocity down to $\log \Delta M / M_{*}=-1$, the point of separation, which is well into the burning layers. However, at $10 \mathrm{Gyr}$, this has not yet reached the surface since at $10^{10} \mathrm{yr}$, a mass loss rate of $10^{-12} M_{\odot} \mathrm{yr}^{-1}$ has only exposed to the surface layers which are above $\sim 10^{-2} M_{*}$. In the same way, mass loss advected all mass above the point of separation, thus explaining the difference in the depths at which Li falls to zero in the diffusion only and $10^{-12} M_{\odot} \mathrm{yr}^{-1}$ models.

Furthermore, the shape of the internal solutions in both sets of models is very different; for $10^{-12} M_{\odot} \mathrm{yr}^{-1}$, VC95 obtain a nearly flat solution from the BSCZ down to Li-burning layers (see their Fig. 4), whereas our solution suggests an important atomic diffusion-induced gradient below the SCZ (see Fig. 8). In order to verify that the difference was not simply due to differences between SCZ masses during evolution, this result was 

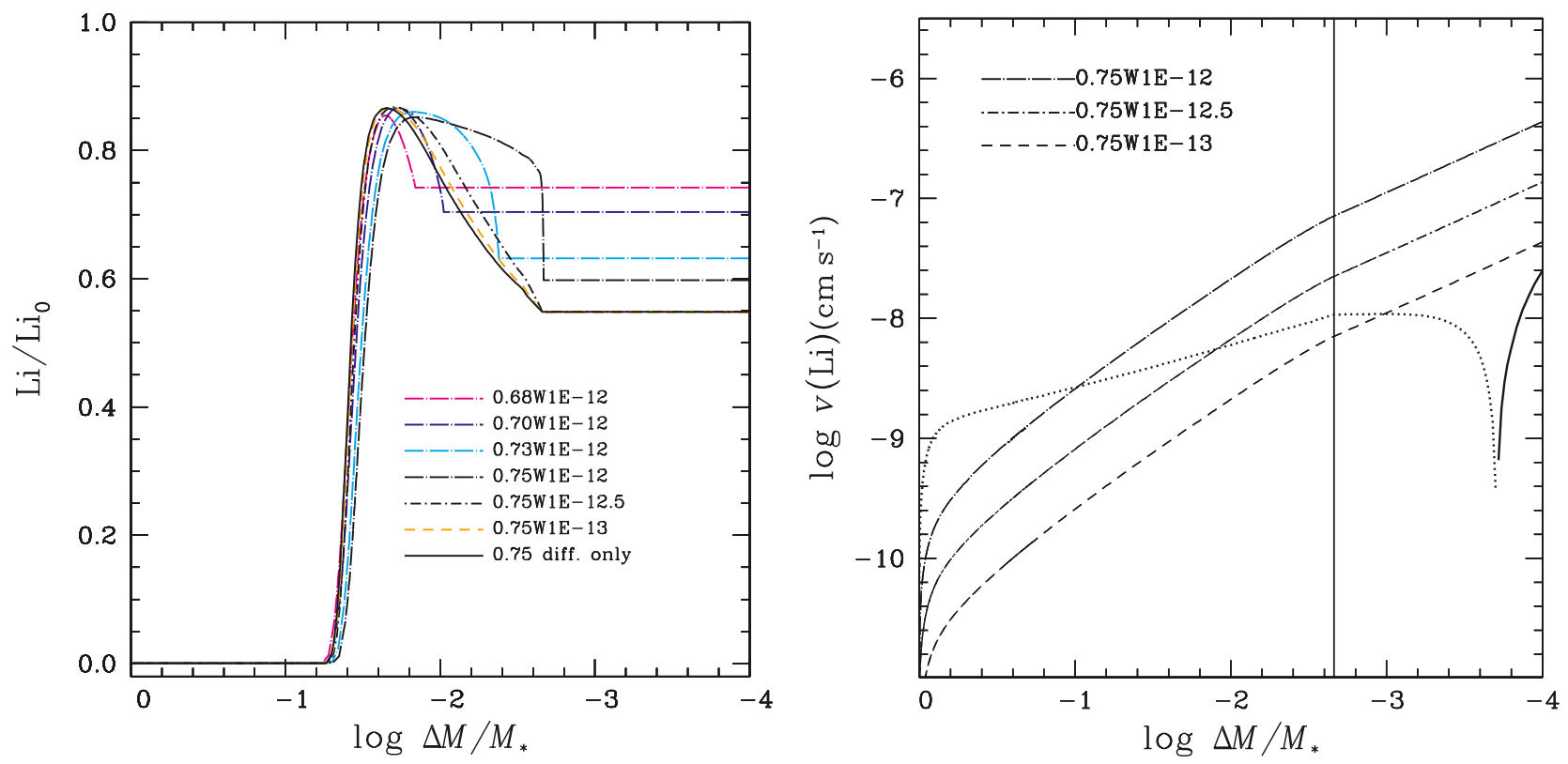

Fig. 8. (Left panel) Internal lithium profiles for $0.75 M_{\odot}$ models with and without mass loss. Three models of different masses were added (colored curves) in order to verify the solution for the $0.75 \mathrm{~W} 1 \mathrm{E}-12$ model. (Right panel) A comparison of diffusion (dotted and solid lines) and wind velocities (dashed lines identified in the figure) for the $0.75 M_{\odot}$ models. The vertical solid line identifies the bottom of the surface convection zone. All models are shown at 10 Gyr. The left panel can be compared to Fig. 4 of Vauclair \& Charbonnel (1995).

compared to smaller mass models with the same mass loss rate (the $0.73 \mathrm{~W} 1 \mathrm{E}-12,0.70 \mathrm{~W} 1 \mathrm{E}-12$ and $0.68 \mathrm{~W} 1 \mathrm{E}-12$ models). Even in models with more massive SCZs, the abundance gradient remains, since it is formed below $\log \Delta M / M_{*} \sim-2$, the point above which the wind has imposed the conservation of flux. The reason for these differences between the two calculations is not evident.

The Li profiles in the left panel of Fig. 8 are understood using the right panel and the assumption of unseparated mass loss from the surface. In the $0.75 M_{\odot}$ diffusion only model, the gradient between $\log \Delta M / M_{*}=-1.8$ and -2.7 is determined mainly by the increasing settling time scale as $\log \Delta M / M_{*}$ increases, that is closer to stellar center. In the $0.75 \mathrm{~W} 1 \mathrm{E}-13$ model the wind only shifts $10^{-3}$ of a solar mass in $10^{10}$ years which only very slightly modifies the profile around $\log \Delta M / M_{*} \sim-2$. In the $0.75 \mathrm{~W} 1 \mathrm{E}-12.5$ model the effect is slightly more pronounced as the mass loss velocity becomes a little larger, in magnitude, than the diffusion velocity as seen in the right panel. In the $0.75 \mathrm{~W} 1 \mathrm{E}-12$ model however the mass loss velocity is larger than the diffusion velocity by a factor of up to six, and flux conservation determines the $\mathrm{Li}$ abundance between $\log \Delta M / M_{*}=-1.8$ and -2.7 as discussed in detail in Sect. 5.1.1 of Vick et al. (2010). Since the mass loss from the surface in unseparated and the outgoing Li concentration is the Li concentration in the convection zone, this implies no separation in the convection zone and the Li mass loss flux is $X(\mathrm{Li}) \rho v_{\text {wind }}$. It is as if the diffusion velocity were zero there. Below the convection zone the Li flux is $\sim X(\mathrm{Li}) \rho\left(v_{\text {wind }}-v_{\mathrm{D}}\right)$. Element conservation at the BSCZ forces a strong gradient just below ${ }^{11}$. It is seen mainly in the $0.75 \mathrm{~W} 1 \mathrm{E}-12$ and $0.73 \mathrm{~W} 1 \mathrm{E}-12$ models. Because the wind velocity hardly exceeds the diffusion velocity below the bottom of

\footnotetext{
${ }^{11}$ More technically, the diffusion term in $v_{\mathrm{D}}$ must compensate for the decrease in $X(\mathrm{Li})$ as one approaches the convection zone. Except where there is a strong $X(\mathrm{Li})$ gradient, only the advective part of $v_{\mathrm{D}}$ plays a significant role. Numerical accuracy required some one hundred zones in the small region of the strong gradient.
}

the $\mathrm{SCZ}$ in the $0.68 M_{\odot}$ model, the gradient is less abrupt in that model.

Finally, while VC95 obtain no effects of chemical separation in all models with $10^{-12} M_{\odot} \mathrm{yr}^{-1}$ (see the caption of their Fig. 4), lithium settling leads to $\sim 0.15$ dex surface abundance reductions (at 12 Gyr) in all our models (see Fig. 7). In terms of isochrone shape, VC95 obtain a monotonous linear increase from the cooler stars to the hotter ones (Figs. 5 and 6 of VC95), while our isochrones dip in the interval $6400 \mathrm{~K} \geq T_{\text {eff }} \geq 6000 \mathrm{~K}$ (Fig. 7).

Some of the differences are related to the difference in models, namely the $\Delta M_{\mathrm{BSCZ}}$ evolution. Other differences could include the way the boundary condition below the SCZ was written and the fact that the wind term in their diffusion equation does not include the often overlooked flux factor $\left(m_{\mathrm{r}} / M_{*}\right)$ which is required in evolutionary models (see Eq. (6) of Paper I). This factor changes $v_{\text {wind }}$ by $1 \%$ at $\log \Delta M / M_{*}=-2$ and by $10 \%$ at $\log \Delta M / M_{*}=-1$. Given that wind and diffusion velocities are comparable for many elements near $\log \Delta M / M_{*}=-1$ (see Figs. 3 and 4), this term modifies central concentrations. For instance, in the $0.779 M_{\odot}$ model with a mass loss rate of $5 \times 10^{-13} M_{\odot} \mathrm{yr}^{-1}$, the central hydrogen concentration decreases below $10^{-3}, \sim 1.3$ Gyr earlier when the correction term is included. The effect on surface concentrations is, however, very small.

\section{Comparison to models with turbulence}

Along with describing the effects of mass loss on Population II evolutionary models with mass loss, this paper's main objective is to contrast models with mass loss to the ones with turbulence presented in Richard et al. (2002b). In particular, one goal is to see if a specific mass loss rate can reproduce the surface abundances exhibited in the T6.09 models ${ }^{9}$ around turnoff, which are compatible with the Spite plateau (Richard et al. 2005).

In Fig. 6, the surface abundances of the 0.80T6.09 and 0.80W1E-12 models are shown to be very similar. Abundances 

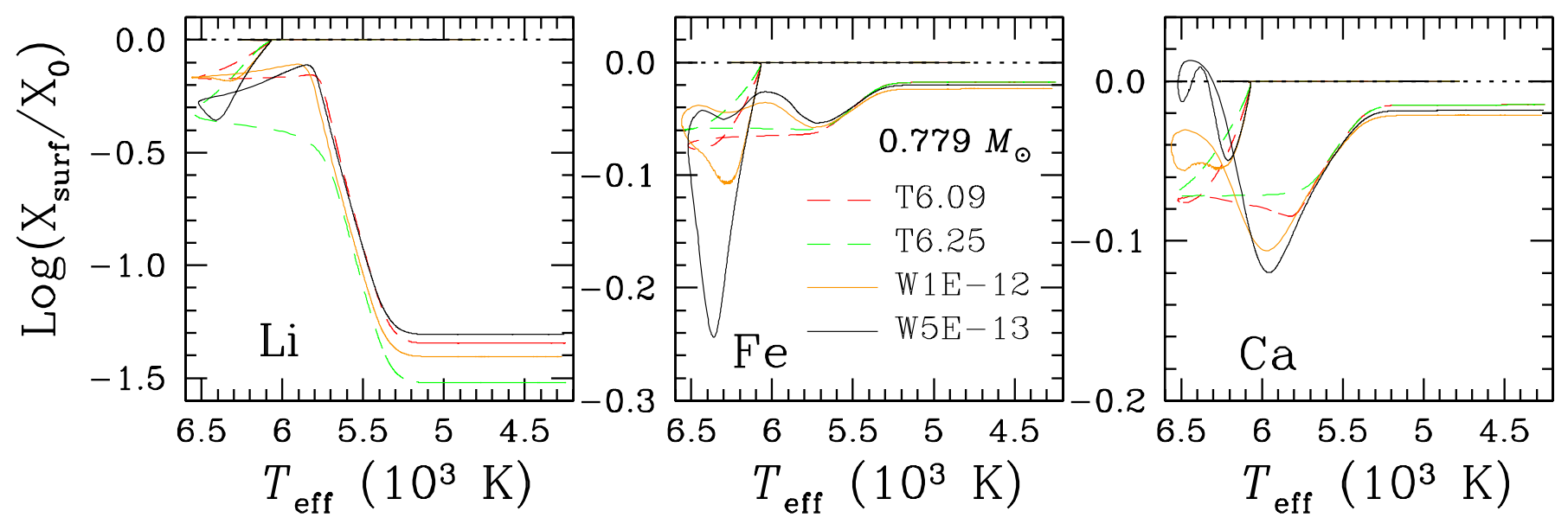

Fig. 9. Evolution of the surface abundances of $\mathrm{Li}, \mathrm{Fe}$, and $\mathrm{Ca}$ as a function of $T_{\text {eff }}$ for two models with turbulence and two with mass loss identified in the figure. The chosen scale emphasizes the behavior on the subgiant and bottom of the giant branch. All models start (pre-main-sequence) to the right of the upper horizontal line and evolve to the left until they reach the main sequence at $T_{\text {eff }} \sim 6100 \mathrm{~K}$. Then, diffusion starts being effective and the $\mathrm{Li}$ abundance, for instance, is reduced by 0.2 to $0.4 \mathrm{dex}$ as the star approaches turnoff, at $T_{\text {eff }} \sim 6600 \mathrm{~K}$. The models then move to the subgiant and giant branches where dilution further reduces the surface $\mathrm{Li}$ abundance by a factor of $\sim 10$. For $\mathrm{Ca}$ and Fe the radiative acceleration complicates the surface abundance evolution in the mass loss cases.

of ${ }^{4} \mathrm{He}$ and ${ }^{7} \mathrm{Li}$ are about 0.02 dex smaller in the model with turbulence, whereas elements with $Z>12$ are on average about $0.03-0.04$ dex smaller (with a maximum of about 0.05 dex for Ar). The differences in surface abundances of elements from $\mathrm{Be}$ to $\mathrm{Mg}$ are smaller than the line width.

In terms of lithium and iron isochrones, as shown in Fig. 7, the differences are just as small. Both types of models lead to nearly flat abundances (above $T_{\text {eff }}=5800 \mathrm{~K}$ ), and their plateau value is nearly the same. At $12 \mathrm{Gyr}$, the maximum difference in Fe abundance, which occurs near $T_{\text {eff }} \simeq 6100 \mathrm{~K}$, is about $0.07 \mathrm{dex}$, though this could be due to the absence of turbulent models in this region. Similarly, for models hotter than $T_{\text {eff }}=5800 \mathrm{~K}$, the maximum difference in ${ }^{7} \mathrm{Li}$ abundances, which occurs at $T_{\text {eff }} \simeq 6200 \mathrm{~K}$, is $0.1 \mathrm{dex}$, and could again be explained by the T6.09 isochrone's poor resolution. Below $T_{\text {eff }}=5800 \mathrm{~K}$, larger differences appear for lithium, likely due to lithium destruction in the model with turbulence. The discrepancy is greater than $0.3 \mathrm{dex}$, although again, the resolution is not sufficient to quantify precisely the difference.

Whereas the surface behavior is quite similar for most models with $\mathrm{T} 6.09$ and $10^{-12} M_{\odot} \mathrm{yr}^{-1}$, the internal solutions are a little more different. By comparing Fig. 3 with Fig. 8 of Richard et al. (2002b), one notes that in the 0.80W1E-12 model, abundances change gradually from the bottom of the SCZ down to about $\log \Delta M / M_{*}=-1$, with most variations between 0.1 and 0.15 dex. However, in the 0.80T6.09 model at turnoff, abundances are constant from the surface down to just be$\operatorname{low} \log \Delta M / M_{*}=-2$. Even though in the mass loss models the abundance variations are relatively small, and the corresponding changes in opacity are likely to be small as well, this could potentially be tested by asteroseismic observations.

\section{Subgiant and giant branches}

The evolution of the surface abundances of $\mathrm{Li}, \mathrm{Fe}$, and $\mathrm{Ca}$ are shown in Fig. 9 for $0.779 M_{\odot}$ models with $Z_{0}=0.00027^{12}$. It is shown as a function of $T_{\text {eff }}$ in order to facilitate comparison with stars on the subgiant and giant branches. At turnoff,

\footnotetext{
12 As is appropriate for NGC 6397. As stated in Sects. 2 and 3, most other models have $Z_{0}=0.00017$.
}

the T6.25 model has approximately the same $\mathrm{Li}$ abundance as the $5 \times 10^{-13} M_{\odot} \mathrm{yr}^{-1}$ model and the T6.09 model approximately the same as the $10^{-12} M_{\odot} \mathrm{yr}^{-1}$ model. Past turnoff, the surface $\mathrm{Li}$ abundance first increases in both models with mass loss (with a maximum at $T_{\text {eff }} \sim 5800 \mathrm{~K}$ ) as the peak in $\mathrm{Li}$ abundance seen at $\log \Delta M / M_{*} \sim-2$ in the left panel of Fig. 8 is mixed to the surface. In the models with turbulence, this Li peak is either reduced (T6.09 model) or eliminated (T6.25 model) as may be seen in Fig. 7 of Richard et al. (2005). In the latter model, the $\mathrm{Li}$ abundance decreases continuously past turnoff to $\sim-1.5$ on the giant branch $\left(T_{\text {eff }}<5000 \mathrm{~K}\right)$. More Li has been destroyed by turbulence in the T6.25 model than carried away by a mass loss rate of $10^{-12} M_{\odot} \mathrm{yr}^{-1}$ since the $\mathrm{Li}$ abundance on the giant branch is smaller in the T6.25 model than in the mass loss one. This implies that turbulence leads to a 0.2 dex additional destruction of $\mathrm{Li}$ in the T6.25 model when compared to models with atomic diffusion alone.

These results may be compared to the calculations and observations of Mucciarelli et al. (2011) and of Nordlander et al. (2012). The T6.25 model leads to an additional 0.2 dex destruction of $\mathrm{Li}$ as appears required for the observations of $\mathrm{Li}$ in lower red giant branch stars (Mucciarelli et al. 2011) to be compatible with WMAP measurements. Neither of the mass loss models leads to as large a reduction of surface $\mathrm{Li}$ on the giant branch as the T6.25 model, so that the RGB observations favor the turbulent T6.25 model. However, one may also compare our results to the Li observations in Figs. 2 and 5 of Nordlander et al. (2012) where isochrones of $\mathrm{Li}$ abundance are compared to observations of NGC 6397. While we do not have isochrones, a comparison is possible using evolutionary variations of $\mathrm{Li}$ abundance since the same turbulent models were used in our calculations and for their Figs. 2 and 5. If one compares the $10^{-12} M_{\odot} \mathrm{yr}^{-1}$ results, to the T6.0 preferred by Nordlander et al. (2012), one notes similar agreement at turnoff, but better agreement on the subgiant branch since maxima in our Fig. 9 appear at a slightly higher $T_{\text {eff }}$ and are more pronounced for the $10^{-12} M_{\odot} \mathrm{yr}^{-1}$ model than for the two turbulent cases. The agreement is also better for the bRGB and RGB observations since the $10^{-12} M_{\odot} \mathrm{yr}^{-1}$ curve is lower than even the T6.09 curve in our Fig. 9. It then appears that models with mass loss lead to at least as good agreement with Li observations as do models with turbulence. 
For $\mathrm{Ca}$ and $\mathrm{Fe}$, the radiative accelerations complicate the evolution in the mass loss cases. For instance, the large $\mathrm{Ca}$ abundance close to the surface for the $11.664 \mathrm{Gyr}$ curve for $\mathrm{Ca}$ on Fig. 3 is caused by the $g_{\mathrm{rad}}(\mathrm{Ca})$ minimum as discussed in relation to that figure. In turn it appears as the abundance maximum at $T_{\text {eff }} \sim 6500 \mathrm{~K}$ in Fig. 9. The Fe local maximum at $T_{\text {eff }} \sim 6000 \mathrm{~K}$ in Fig. 9 is similarly caused by the $g_{\mathrm{rad}}(\mathrm{Fe}) \mathrm{min}-$ imum for the 11.664 Gyr curve at $\log \Delta M / M_{*} \sim-3$ in Fig. 3. These properties affect the comparison to observations for the mass loss models. The comparison is shown for the turbulent models in Fig. 2 of Nordlander et al. (2012). If one assumes the abundance shifts just discussed, the agreement with observations would be poorer with the mass loss models for both $\mathrm{Fe}$ and $\mathrm{Ca}$ at $T_{\text {eff }} \sim 6400 \mathrm{~K}$ while at $T_{\text {eff }} \sim 6000 \mathrm{~K}$, it would be poorer for $\mathrm{Fe}$ and unchanged for $\mathrm{Ca}$.

Given the difficulty justifying such a high mass loss rate we did not push the comparison with observations further.

\section{Conclusion}

Evolutionary models with atomic diffusion and unseparated mass loss explain some characteristics observed in galactic halo dwarfs. A mass loss rate of $5 \times 10^{-13} M_{\odot} \mathrm{yr}^{-1}$ leads to surface lithium isochrones that exhibit plateau-like behavior with a maximum scatter of about 0.1 dex around an average value that is -0.3 dex below the initial lithium abundance (Fig. 7). For models with mass loss rates of $10^{-12} M_{\odot} \mathrm{yr}^{-1}$, the lithium isochrones are even flatter with a scatter of about 0.05 dex around an average value about -0.2 dex lower than the initial abundance.

While mass loss does not have a significant impact on a star's structural properties, its effects on abundances, both in the interior and on the surface, can be important. In the interior, abundance profiles are either dominated by atomic diffusion - and reflect local variations in $g_{\mathrm{rad}}$ - or are dominated by the wind - and adjust in order to conserve the flux coming from below (Sect. 3.1). A mass loss rate of $10^{-13} M_{\odot} \mathrm{yr}^{-1}$ reduces both internal and surface anomalies by up to a factor of 6-7, while a mass loss rate of $10^{-12} M_{\odot} \mathrm{yr}^{-1}$ reduces all anomalies to below $0.2 \mathrm{dex}$ (Fig. 4 and 6). While the $10^{-12} M_{\odot} \mathrm{yr}^{-1}$ models lead to generalized underabundances for all elements, lower mass loss rates allow for overabundances to develop at the surface.

The mass loss models presented in this paper differ significantly from those presented in Vauclair \& Charbonnel (1995) (Fig. 8). Although some of this difference can be attributed to differences in input parameters, other discrepancies are more difficult to explain (Sect. 4). In one critical aspect however, the results are robust: both calculations conclude that below $10^{-14} M_{\odot} \mathrm{yr}^{-1}$ mass loss has virtually no effect while above $10^{-12} M_{\odot} \mathrm{yr}^{-1}$ it eliminates all effects of atomic diffusion from the surface.

With respect to the T6.09 models from Richard et al. (2002b), the models with a mass loss rate of $10^{-12} M_{\odot} \mathrm{yr}^{-1}$ are very similar above $T_{\text {eff }}=5800 \mathrm{~K}$ (Figs. 6 and 7). Below this temperature, turbulence leads to greater lithium destruction. Because of differences in the internal distribution of elements (Sect. 5), asteroseismology could perhaps distinguish between the two scenarios.

Unfortunately, even for mass loss rates of $10^{-12} M_{\odot} \mathrm{yr}^{-1}$, the implied stellar winds are barely detectable through direct observation. Furthermore, considering that acceleration processes for these types of winds are unknown, it is difficult to justify mass loss rates that are 50 times higher than the solar mass loss rate $\left(\sim 2 \times 10^{-14} M_{\odot} \mathrm{yr}^{-1}\right)$. It is unlikely that winds in Population II stars are driven by radiation; therefore, if these stars host solarlike winds, one could hope to observe solar-like coronae and/or $\mathrm{X}$-ray emission.

A signature of a chromosphere has recently been observed in Pop II dwarfs: the He line $10830 \mathrm{~A}$ is present in almost all dwarfs with $[\mathrm{Fe} / \mathrm{H}]=-0.5$ to -3.7 (Takeda \& Takada-Hidai 2011). Signs of chromospheres had earlier been reported in these stars by Peterson \& Schrijver (2001). On the other hand, Cranmer \& Saar (2011) have recently evaluated mass loss rates in various types of stars. According to their Fig. 13, mass loss rates of $\sim 10^{-12} M_{\odot} \mathrm{yr}^{-1}$ are to be expected in dwarf stars of $T_{\mathrm{eff}} \sim$ $6000 \mathrm{~K}$ of solar metallicity that have rotation periods of around six days. They do not give equivalent values for lower metallicity stars. The most critical factor is, however, the rotation rate. Pop II dwarfs are believed to rotate slowly. However, there are observations (reviewed in Fig. 6 of Takeda \& Takada-Hidai 2011) suggesting that many rotate at $v_{e} \sin i \sim 5 \mathrm{~km} \mathrm{~s}^{-1}$. It would seem important to confirm both the rotation rate observations and expected mass loss rates for low metallicity stars. In the meantime it seems premature to disregard mass loss as a process competing with atomic diffusion in Pop II stars.

As a next step, these models could be extended to the $\mathrm{He}$ flash in order to further constrain the effects of mass loss in Population II giants, for which mass loss has extensively been observed and studied (Origlia et al. 2007). It would also be interesting to see if such mass loss rates would affect, or even eliminate, the effects of atomic diffusion (Michaud et al. 2010) and/or of thermohaline mixing (Charbonnel \& Zahn 2007; Traxler et al. 2011).

Acknowledgements. M. Vick would like to thank the Département de physique de l'Université de Montréal for financial support, along with everyone at the GRAAL in Montpellier for their amazing hospitality. We acknowledge the financial support of Programme National de Physique Stellaire (PNPS) of CNRS/INSU, France. This research was partially supported by NSERC at the Université de Montréal. Finally, we thank Calcul Québec for providing us with the computational resources required for this work. We thank an anonymous referee for useful comments.

\section{References}

Aoki, W., Barklem, P. S., Beers, T. C., et al. 2009, ApJ, 698, 1803

Asplund, M., Lambert, D. L., Nissen, P. E., Primas, F., \& Smith, V. V. 2006, ApJ, 644,229

Böhm-Vitense, E. 1992, Introduction to Stellar Astrophysics (Cambridge, UK: Cambridge University Press), 3

Bonifacio, P. 2002, A\&A, 395, 515

Bonifacio, P., Molaro, P., Sivarani, T., et al. 2007, A\&A, 462, 851

Cayrel, R., Steffen, M., Chand, H., et al. 2007, A\&A, 473, L37

Charbonnel, C., \& Primas, F. 2005, A\&A, 442, 961

Charbonnel, C., \& Talon, S. 2005, Science, 309, 2189

Charbonnel, C., \& Zahn, J. 2007, A\&A, 467, L15

Charbonnel, C., Vauclair, S., \& Zahn, J. 1992, A\&A, 255, 191

Coc, A., Vangioni-Flam, E., Descouvemont, P., Adahchour, A., \& Angulo, C. 2004, ApJ, 600, 544

Cranmer, S. R., \& Saar, S. H. 2011, ApJ, 741, 54

Cumberbatch, D., Ichikawa, K., Kawasaki, M., et al. 2007, Phys. Rev. D, 76, 123005

Cyburt, R. H., Fields, B. D., \& Olive, K. A. 2003, Phys. Lett. B, 567, 227

Cyburt, R. H., Fields, B. D., \& Olive, K. A. 2008, J. Cosmol. Astro-Part. Phys., 11,12

Deliyannis, C. P., Demarque, P., \& Kawaler, S. D. 1990, ApJS, 73, 21

Gonzalez, J.-F., LeBlanc, F., Artru, M.-C., \& Michaud, G. 1995, ApJ, 297, 223

González Hernández, J. I., Bonifacio, P., Caffau, E., et al. 2009, A\&A, 505, L13 Ichikawa, K., Kawasaki, M., \& Takahashi, F. 2004, Phys. Lett. B, 597, 1

Iglesias, C. A., \& Rogers, F. 1996, ApJ, 464, 943

Korn, A. J., Grundahl, F., Richard, O., et al. 2006, Nat, 442, 657

Korn, A. J., Grundahl, F., Richard, O., et al. 2007, ApJ, 671, 402

Krishna-Swamy, K. S. 1966, ApJ, 145, 176

LeBlanc, F., Michaud, G., \& Richer, J. 2000, ApJ, 538, 876 
Lind, K., Primas, F., Charbonnel, C., Grundahl, F., \& Asplund, M. 2009, A\&A, 503,545

Lind, K., Asplund, M., Collet, R., \& Meléndez, J. 2012, Mem. Soc. Astron. It. Supp., 22, 142

Meléndez, J., \& Ramírez, I. 2004, ApJ, 615, L33

Meléndez, J., Casagrande, L., Ramírez, I., Asplund, M., \& Schuster, W. J. 2010, A\&A, 515, L3

Michaud, G., Fontaine, G., \& Beaudet, G. 1984, ApJ, 282, 206

Michaud, G., Richer, J., \& Richard, O. 2007, ApJ, 670, 1178

Michaud, G., Richer, J., \& Richard, O. 2008, ApJ, 675, 1223

Michaud, G., Richer, J., \& Richard, O. 2010, A\&A, 510, A104

Michaud, G., Richer, J., \& Vick, M. 2011, A\&A, 534, A18

Mucciarelli, A., Salaris, M., Lovisi, L., et al. 2011, MNRAS, 412, 81

Nordlander, T., Korn, A. J., Richard, O., \& Lind, K. 2012, ApJ, 753, 48

Origlia, L., Rood, R. T., Fabbri, S., et al. 2007, ApJ, 667, L85

Peterson, R. C. \& Schrijver, C. J. 2001, in 11th Cambridge Workshop on Cool Stars, Stellar Systems and the Sun, eds. R. J. Garcia Lopez, R. Rebolo, \& M. R. Zapaterio Osorio, ASP Conf. Ser., 223, 300

Piau, L., Beers, T. C., Balsara, D. S., et al. 2006, ApJ, 653, 300

Pinsonneault, M. H., Walker, T. P., Steigman, G., \& Narayanan, V. K. 1999, ApJ, 527,180

Profitt, C. R., \& Michaud, G. 1991, ApJ, 371, 584

Regis, M. \& Clarkson, C. 2012, Gen. Relativ. Grav., 44, 567

Richard, O., Michaud, G., \& Richer, J. 2001, ApJ, 558, 377

Richard, O., Michaud, G., \& Richer, J. 2002a, ApJ, 580, 1100

Richard, O., Michaud, G., Richer, J., et al. 2002b, ApJ, 568, 979

Richard, O., Michaud, G., \& Richer, J. 2005, ApJ, 619, 538
Richer, J., Michaud, G., Rogers, F., Turcotte, S., \& Iglesias, C. A. 1998, ApJ, 492,833

Richer, J., Michaud, G., \& Turcotte, S. 2000, ApJ, 529, 338

Ryan, S. G., Norris, J. E., \& Beers, T. C. 1999, ApJ, 523, 654

Salaris, M., Groenewegen, M. A. T., \& Weiss, A. 2000, A\&A, 355, 299

Sbordone, L., Bonifacio, P., Caffau, E., et al. 2010, A\&A, 522, A26

Sbordone, L., Bonifacio, P., \& Caffau, E. 2012, Mem. Soc. Astron. It. Supp., 22, 29

Spergel, D. N., Bean, R., Doré, O., et al. 2007, ApJS, 170, 377

Spite, F., \& Spite, M. 1982, A\&A, 115, 357

Spite, M., Spite, F., \& Maillard, J. P. 1984, A\&A, 141, 56

Steigman, G. 2007, Ann. Rev. Nucl. Part. Sci., 57, 463

Takeda, Y., \& Takada-Hidai, M. 2011, PASJ, 63, 547

Talon, S., \& Charbonnel, C. 2004, A\&A, 418, 1051

Traxler, A., Garaud, P., \& Stellmach, S. 2011, ApJ, 728, L29

Turcotte, S., Richer, J., Michaud, G., Iglesias, C. A., \& Rogers, F. 1998, ApJ, 504,539

VandenBerg, D. A., Bolte, M., \& Stetsson, P. B. 1996, ARA\&A, 34, 461

VandenBerg, D. A., Swenson, F. J., Rogers, F. J., Iglesias, C. A., \& Alexander, D. R. 2000, ApJ, 532, 430

VandenBerg, D. A., Richard, O., Michaud, G., \& Richer, J. 2002, ApJ, 571, 487 VandenBerg, D. A., Edvardsson, B., Eriksson, K., \& Gustafsson, B. 2008, ApJ, 675,746

Vauclair, S. 1988, ApJ, 335, 971

Vauclair, S., \& Charbonnel, C. 1995, A\&A, 295, 715

Vick, M., Michaud, G., Richer, J., \& Richard, O. 2010, A\&A, 521, A62

Vick, M., Michaud, G., Richer, J., \& Richard, O. 2011, A\&A, 526, A37 
M. Vick et al.: Population II stars and the Spite plateau

\section{Appendix A: Version of Figs. 3 and 4 with all atomic species included in the calculations}

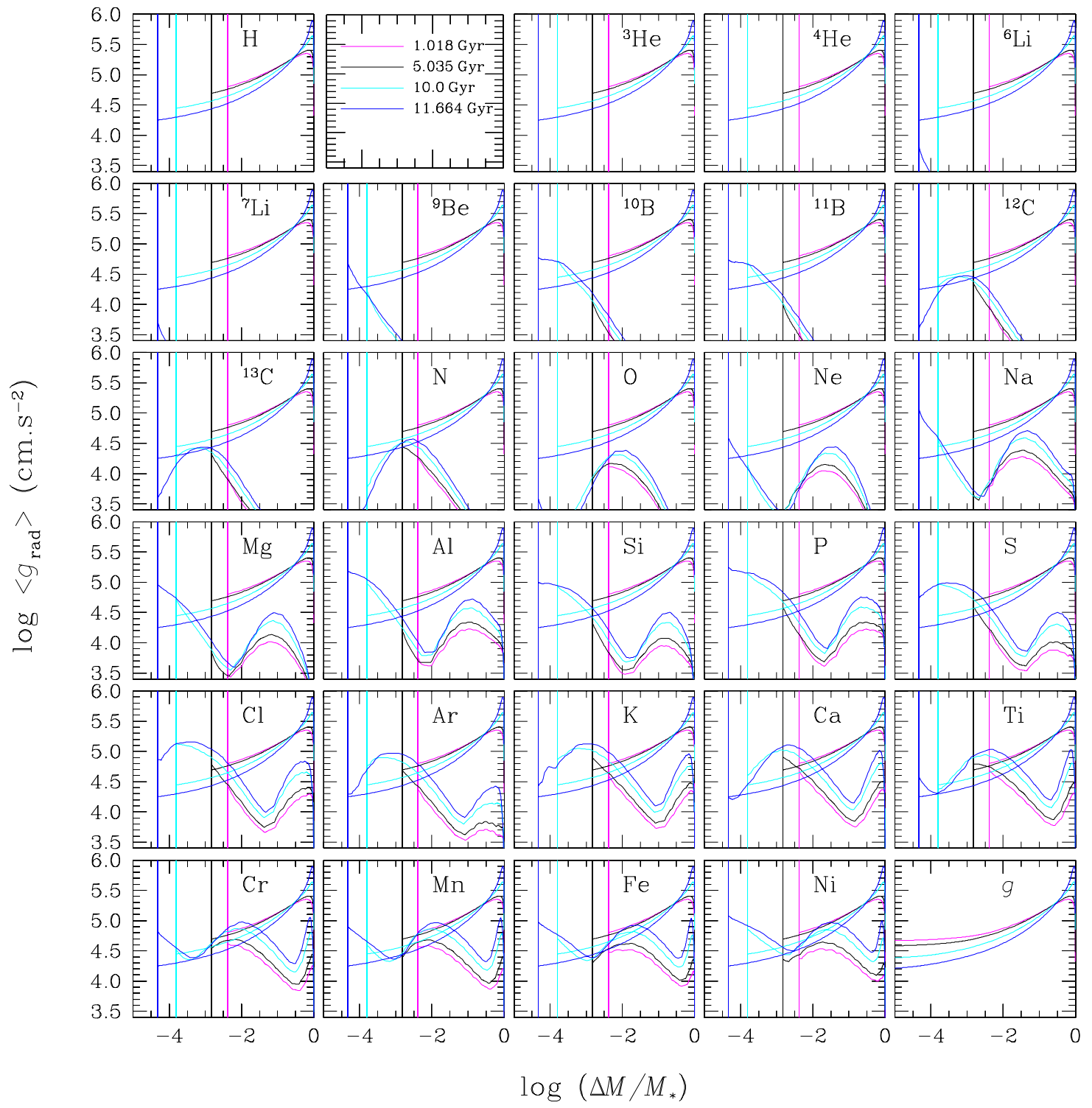

Fig. A.1. Radiative accelerations and gravity at four different ages in a $0.8 M_{\odot}$ model with $\dot{M}=10^{-12} M_{\odot} \mathrm{yr}^{-1}$. The vertical lines indicate the position of the bottom of the SCZ. The ages are identified in the figure. 
A\&A 552, A131 (2013)

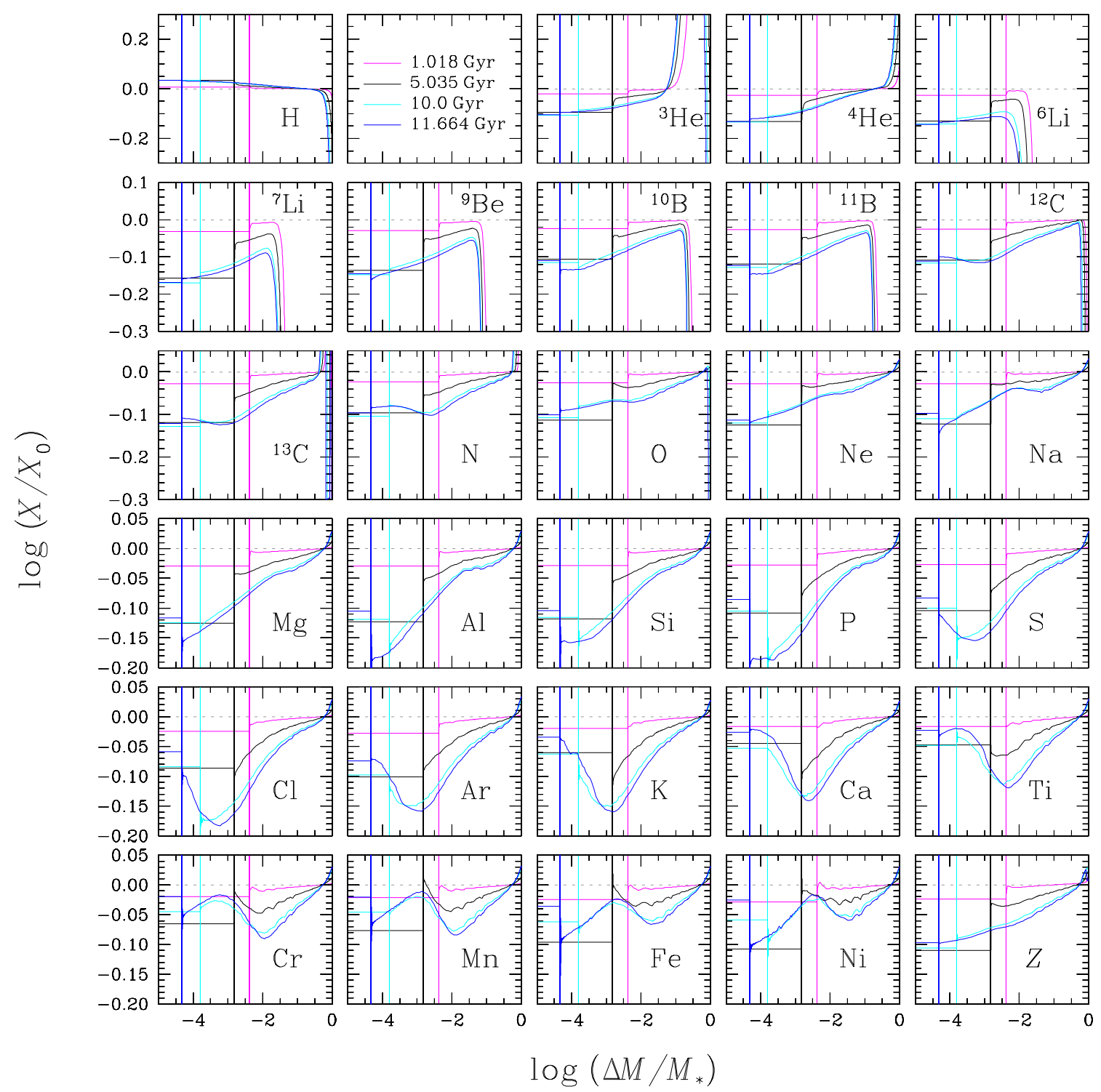

Fig. A.2. Abundance profiles at four different ages in a $0.8 M_{\odot}$ model with $\dot{M}=10^{-12} M_{\odot} \mathrm{yr}^{-1}$ and an initial metallicty of $Z_{0}=0.00017$. The vertical lines indicate the position of the bottom of the SCZ. The ages are identified in the figure. 
M. Vick et al.: Population II stars and the Spite plateau

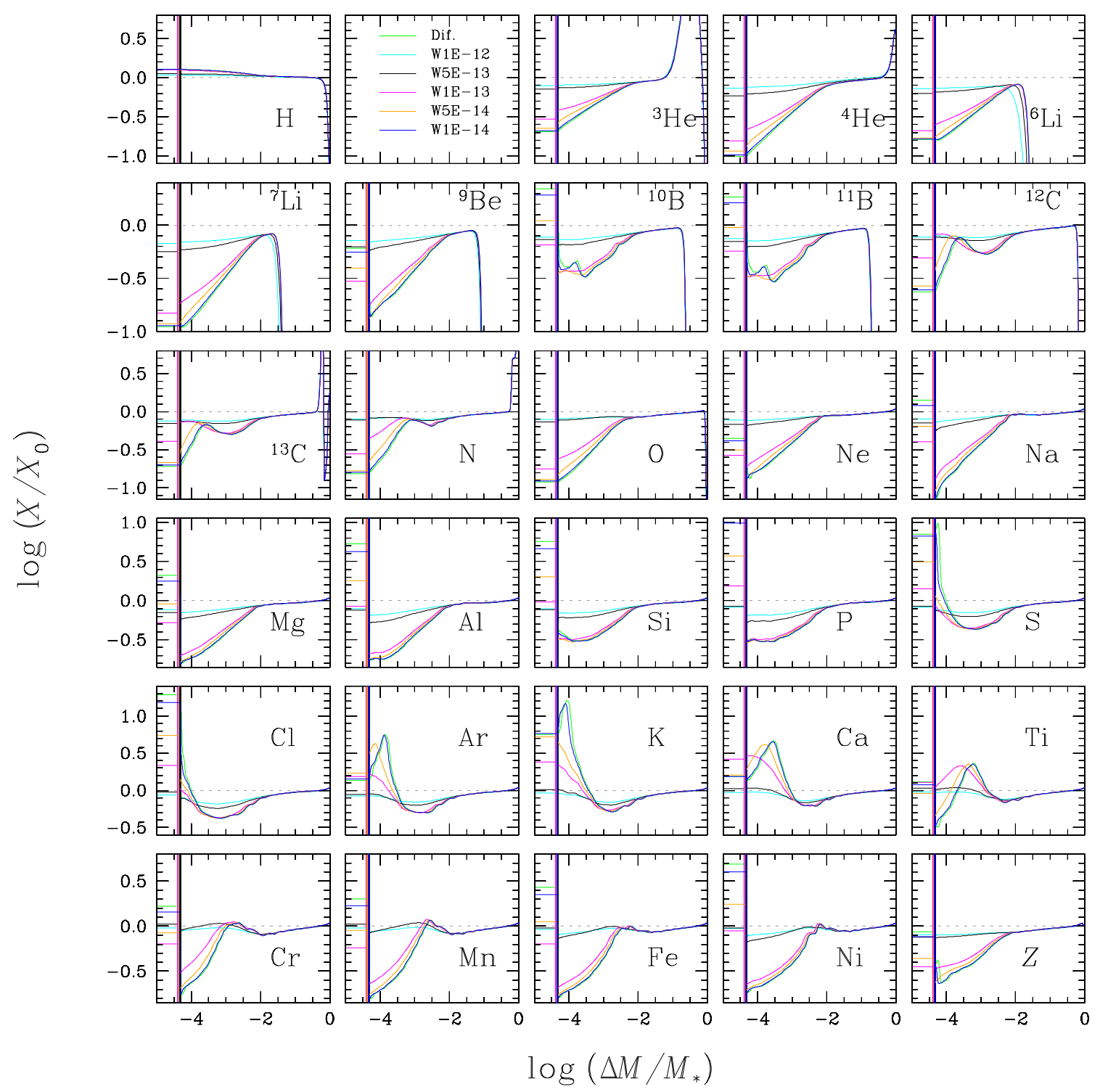

Fig. A.3. Abundance profiles for the model without mass loss and for mass loss rates, from $\dot{M}=10^{-14} M_{\odot} \mathrm{yr}^{-1}$ to $\dot{M}=10^{-12} M_{\odot} \mathrm{yr}^{-1}$ in a $0.8 M_{\odot}$ model and an initial metallicty of $Z_{0}=0.00017$. The internal concentration profiles are shown just before turnoff near $11.5 \mathrm{Gyr}$. The vertical lines indicate the position of the bottom of the SCZ. The mass loss rates are identified in the figure. 
A\&A 552, A131 (2013)

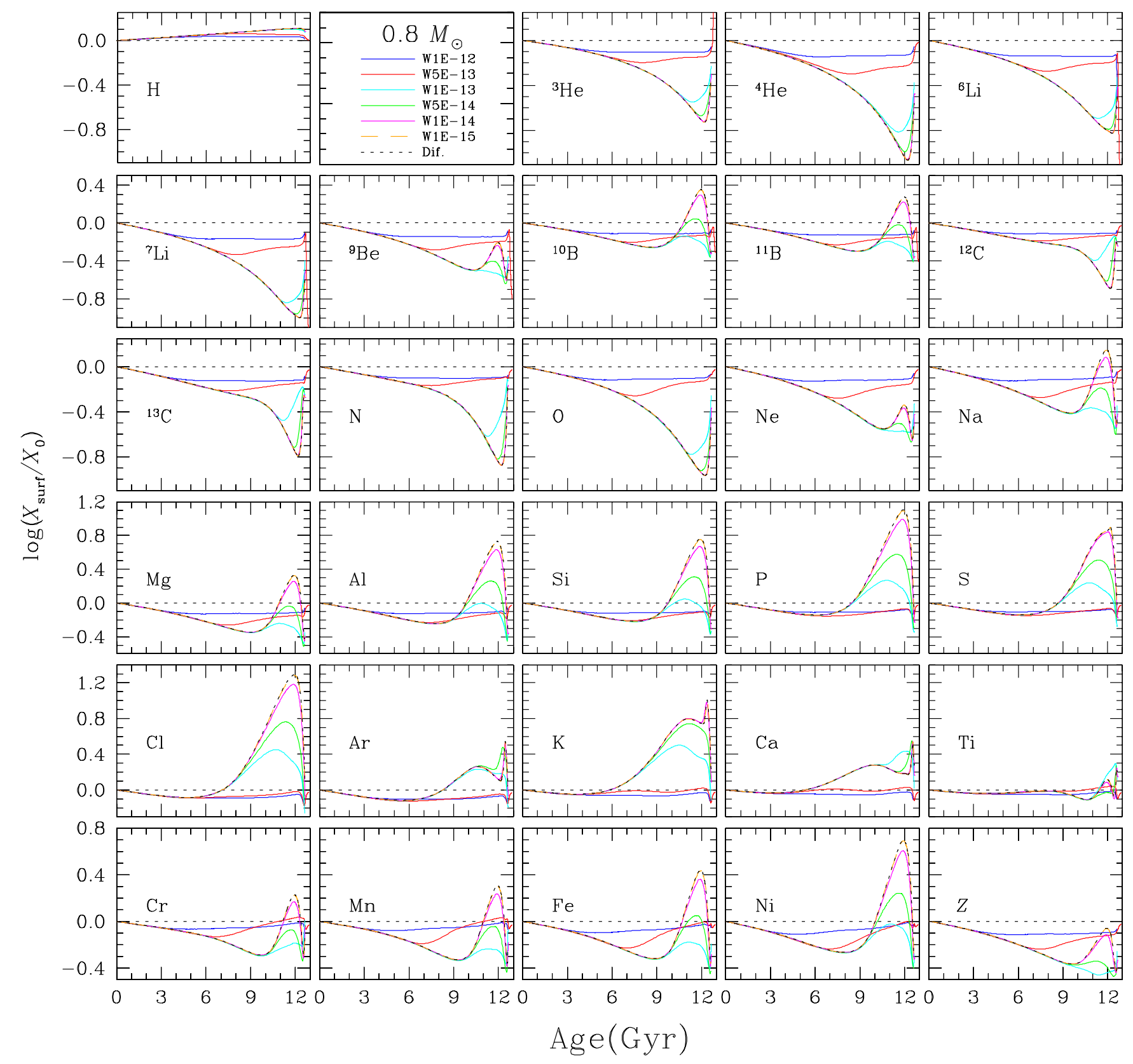

Fig. A.4. Evolution of surface abundances for the model without mass loss (dotted line) and for mass loss rates, from $\dot{M}=10^{-15} M_{\odot} \mathrm{yr}^{-1}$, to $\dot{M}=10^{-12} M_{\odot} \mathrm{yr}^{-1}$ in a $0.8 M_{\odot}$ model and an initial metallicty of $Z_{0}=0.00017$. One cannot distinguish the curves for the model without mass loss from those for the model with $\dot{M}=10^{-15} M_{\odot} \mathrm{yr}^{-1}$. The mass loss rates are identified in the figure. 